\title{
Lattice-Based Group Signatures: Achieving Full Dynamicity (and Deniability) with Ease
}

\author{
San Ling, Khoa Nguyen, Huaxiong Wang, Yanhong Xu \\ Division of Mathematical Sciences, \\ School of Physical and Mathematical Sciences, \\ Nanyang Technological University, Singapore. \\ \{lingsan, khoantt, hxwang, xu0014ng\}@ntu.edu.sg
}

\begin{abstract}
Lattice-based group signature is an active research topic in recent years. Since the pioneering work by Gordon, Katz and Vaikuntanathan (Asiacrypt 2010), eight other schemes have been proposed, providing various improvements in terms of security, efficiency and functionality. However, most of the existing constructions work only in the static setting where the group population is fixed at the setup phase. The only two exceptions are the schemes by Langlois et al. (PKC 2014) that handles user revocations (but new users cannot join), and by Libert et al. (Asiacrypt 2016) which addresses the orthogonal problem of dynamic user enrollments (but users cannot be revoked).

In this work, we provide the first lattice-based group signature that offers full dynamicity (i.e., users have the flexibility in joining and leaving the group), and thus, resolve a prominent open problem posed by previous works. Moreover, we achieve this non-trivial feat in a relatively simple manner. Starting with Libert et al.'s fully static construction (Eurocrypt 2016) - which is arguably the most efficient lattice-based group signature to date, we introduce simple-but-insightful tweaks that allow to upgrade it directly into the fully dynamic setting. More startlingly, our scheme even produces slightly shorter signatures than the former, thanks to an adaptation of a technique proposed by Ling et al. (PKC 2013), allowing to prove inequalities in zero-knowledge. The scheme satisfies the strong security requirements of Bootle et al.'s model (ACNS 2016), under the Short Integer Solution (SIS) and the Learning With Errors (LWE) assumptions.

Furthermore, we demonstrate how to equip the obtained group signature scheme with the deniability functionality in a simple way. This attractive functionality, put forward by Ishida et al. (CANS 2016), enables the tracing authority to provide an evidence that a given user is not the owner of a signature in question. In the process, we design a zero-knowledge protocol for proving that a given LWE ciphertext does not decrypt to a particular message.
\end{abstract}

Keywords. lattice-based group signatures; full dynamicity; updatable Merkle trees; deniability 


\section{Introduction}

Group signature, introduced by Chaum and van Heyst [15], is a fundamental anonymity primitive which allows members of a group to sign messages on behalf of the whole group. Yet, users are kept accountable for the signatures they issue since a tracing authority can identify them should the need arise. There have been numerous works on group signatures in the last quarter-century.

Ateniese et al. 2 proposed the first scalable instantiation meeting the security properties that can be intuitively expected from the primitive, although clean security notions were not available yet at that time. Bellare et al. 44 filled this gap by providing strong security notions for static groups, in which the group population is fixed at the setup phase. Subsequently, Kiayias and Yung 25] and Bellare et al. [5] established the models capturing the partially dynamic setting, where users are able to join the group at any time, but once they have done so, they cannot leave the group. Sakai et al. [47] strengthened these models by suggesting the notion of opening soundness, guaranteeing that a valid signature only traces to one user. Efficient schemes satisfying these models have been proposed in the random oracle model [4317] and in the standard model [21/34].

One essential functionality of group signatures is the support for membership revocation. Enabling this feature in an efficient manner is quite challenging, since one has to ensure that revoked users are no longer able to sign messages and the workloads of other parties (managers, non-revoked users, verifiers) do not significantly increase in the meantime. Several different approaches have been suggested [1012 50426] to address this problem, and notable pairing-based constructions supporting both dynamic joining and efficient revocation were given in 403332 . Very recently, Bootle et al. 7] pointed out a few shortcomings of previous models, and put forward stringent security notions for fully dynamic group signatures. They also demonstrated a construction satisfying these notions based on the decisional Diffie-Hellman (DDH) assumption, following a generic transformation from a secure accountable ring signature scheme 8 .

Another interesting functionality of group signatures that we consider in this work is deniability, suggested by Ishida et al. 22. It allows the tracing authority to provide a digital evidence that a given group user did not generate a signature in question. In [22], Ishida et al. discussed various situations in which such functionality helps to protect the privacy of users. For instance, suppose that the entrace/exit control of a building is implemented using a group signature scheme, and the police wants to check whether a suspect was in this building at the time of a crime. If the police asks the tracing authority to reveal the signers of all signatures generated during that time period, then this will seriously violate the privacy of innocent users. In such situation, deniable group signatures make it possible to prove that the signer of a given signature is not the suspect, while still keeping the signer's identity secret. As shown by Ishida et al. 22, the main technical challenge towards realizing the deniability functionality consists of constructing a zero-knowledge proof/argument that a given ciphertext does not decrypt to a particular message. Such a mechanism is non-trivial to real- 
ize in general, but Ishida et al. 22] managed to achieve it from pairing-based assumptions.

For the time being, existing group signature schemes offering full dynamicity and/or deniability all rely on number-theoretic assumptions which are vulnerable to quantum attacks [48. To avoid putting all eggs in one basket, it is thus encouraging to consider instantiations based on alternative, post-quantum foundations, e.g., lattice assumptions. In view of this, let us now look at the topic of lattice-based group signatures.

LATTICE-BASED GROUP SIGNATURES. Lattice-based cryptography has been an exciting research area since the seminal works of Regev [45] and Gentry et al. [19]. Along with other primitives, lattice-based group signature has received noticeable attention in recent years. The first scheme was introduced by Gordon, Katz and Vaikuntanathan 20 whose solution produced signature size linear in the number of group users $N$. Camenisch et al. [13] then extended [20] to achieve anonymity in the strongest sense. Later, Laguillaumie et al. 26] put forward the first scheme with the signature size logarithmic in $N$, at the cost of relatively large parameters. Simpler and more efficient solutions with $\mathcal{O}(\log N)$ signature size were subsequently given by Nguyen et al. 444 and Ling et al. [36. Libert et al. [30] obtained substantial efficiency improvements via a construction based on Merkle trees which eliminates the need for GPV trapdoors [19]. More recently, a scheme supporting message-dependent opening (MDO) feature [46] was proposed in [31. All the schemes mentioned above are designed for static groups.

The only two known lattice-based group signatures that have certain dynamic features were proposed by Langlois et al. 27] and Libert et al. 28. The former is a scheme with verifier-local revocation (VLR) [6], which means that only the verifiers need to download the up-to-date group information. The latter addresses the orthogonal problem of dynamic user enrollments (but users cannot be revoked). To achieve those partially dynamic functionalities, both of the proposals have to incorporate relatively complicated mechanisms 1 and both heavily rely on lattice trapdoors.

The discussed above situation is somewhat unsatisfactory, given that the full dynamicity feature is highly desirable in most applications of group signatures (e.g., protecting the privacy of commuters in public transportation), and it has been achieved based on number-theoretic assumptions. This motivates us to work on fully dynamic group signatures from lattices. Besides, considering that the journey to partial dynamicity in previous works [27/28] was shown not easy, we ask ourselves an inspiring question: Can we achieve full dynamicity with ease? Furthermore, given that deniability is an attractive and non-trivial functionality [22, can we also realize it with ease in the context of lattice-based group signatures? At the end of the day, it is good to solve an open research

\footnotetext{
${ }^{1}$ Langlois et al. considered users' "tokens" as functions of Bonsai signatures [14 and associated them with a sophisticated revocation technique, while Libert et al. used a variant of Boyen's signature [9] to sign users' public keys. Both underlying signature schemes require long keys and lattice trapdoors.
} 
question, but it would be even better and more exciting to do this in a simple way. To make it possible, we will likely need some new and insightful ideas.

Our Results And Techniques. Our main contribution is the first fully dynamic group signature from lattices. The scheme satisfies the strong security requirements put forward by Bootle et al. 7, under the Short Integer Solution (SIS) and the Learning With Errors (LWE) assumptions. As in all previous lattice-based group signatures, our scheme is analyzed in the random oracle model. Additionally, we incorporate the deniability functionality suggested by Ishida et al. 22] into Bootle et al.'s model, and then demonstrate how to make our fully dynamic group signature scheme deniable.

\begin{tabular}{|c|c|c|c|c|c|c|c|}
\hline Scheme & $\begin{array}{l}\text { Sig. } \\
\text { size }\end{array}$ & $\begin{array}{c}\text { Group } \\
\text { PK size }\end{array}$ & \begin{tabular}{|l|} 
Signer's \\
SK size \\
\end{tabular} & \begin{tabular}{|l|} 
Trap- \\
door?
\end{tabular} & Model & $\begin{array}{r}\text { Extra info } \\
\text { per epoch }\end{array}$ & \begin{tabular}{|l} 
Denia- \\
bility
\end{tabular} \\
\hline GKV [20] & $\widetilde{\mathcal{O}}\left(\lambda^{2} \cdot N\right)$ & $\widetilde{\mathcal{O}}\left(\lambda^{2} \cdot N\right)$ & $\widetilde{\mathcal{O}}\left(\lambda^{2}\right)$ & yes & static & NA & $?$ \\
\hline CNR 13 & $\widetilde{\mathcal{O}}\left(\lambda^{2} \cdot N\right)$ & $\widetilde{\mathcal{O}}\left(\lambda^{2}\right)$ & $\widetilde{\mathcal{O}}\left(\lambda^{2}\right)$ & yes & static & NA & $?$ \\
\hline LLLS [26] & $\widetilde{\mathcal{O}}(\lambda \cdot \ell)$ & $\mathcal{O}\left(\lambda^{2} \cdot \ell\right)$ & $\widetilde{\mathcal{O}}\left(\lambda^{2}\right)$ & yes & static & $\mathrm{NA}$ & $?$ \\
\hline \multirow{2}{*}{ LLNW 27] } & \multirow{2}{*}{$\widetilde{\mathcal{O}}(\lambda \cdot \ell)$} & \multirow{2}{*}{$\widetilde{\mathcal{O}}\left(\lambda^{2} \cdot \ell\right)$} & \multirow{2}{*}{$\widetilde{\mathcal{O}}(\lambda \cdot \ell)$} & \multirow{2}{*}{ yes } & \multirow{2}{*}{ VLR } & Sign: no & \multirow{2}{*}{$?$} \\
\hline & & & & & & $\overline{\text { Ver: } \widetilde{\mathcal{O}}(\lambda) \cdot R}$ & \\
\hline NZZ [44] & $\widetilde{\mathcal{O}}\left(\lambda+\ell^{2}\right)$ & $\widetilde{\mathcal{O}}\left(\lambda^{2} \cdot \ell^{2}\right)$ & $\widetilde{\mathcal{O}}\left(\lambda^{2}\right)$ & yes & static & NA & $?$ \\
\hline LNW 36] & $\widetilde{\mathcal{O}}(\lambda \cdot \ell)$ & $\widetilde{\mathcal{O}}\left(\lambda^{2} \cdot \ell\right)$ & $\widetilde{\mathcal{O}}(\lambda)$ & yes & static & NA & $\star$ \\
\hline LLNW 30 & $\widetilde{\mathcal{O}}(\lambda \cdot \ell)$ & $\widetilde{\mathcal{O}}\left(\lambda^{2}+\lambda \cdot \ell\right)$ & $\widetilde{\mathcal{O}}(\lambda \cdot \ell)$ & FREE & static & NA & $\star$ \\
\hline $\mathrm{LLM}+28$ & $\widetilde{\mathcal{O}}(\lambda \cdot \ell)$ & $\widetilde{\mathcal{O}}\left(\lambda^{2} \cdot \ell\right)$ & $\widetilde{\mathcal{O}}(\lambda)$ & yes & $\begin{array}{l}\text { partially } \\
\text { dynamic }\end{array}$ & NA & $\star$ \\
\hline LMN 31] & $\widetilde{\mathcal{O}}(\lambda \cdot \ell)$ & $\widetilde{\mathcal{O}}\left(\lambda^{2} \cdot \ell\right)$ & $\widetilde{\mathcal{O}}(\lambda)$ & yes & $\mathrm{MDO}$ & NA & $?$ \\
\hline \multirow[t]{2}{*}{ Ours } & \multirow[t]{2}{*}{$\widetilde{\mathcal{O}}(\lambda \cdot \ell)$} & \multirow[t]{2}{*}{$\widetilde{\mathcal{O}}\left(\lambda^{2}+\lambda \cdot \ell\right)$} & \multirow[t]{2}{*}{$\widetilde{\mathcal{O}}(\lambda)+\ell$} & \multirow[t]{2}{*}{ FREE } & \multirow{2}{*}{$\begin{array}{c}\text { fully } \\
\text { dynamic }\end{array}$} & $\begin{array}{l}\text { Sign: } \\
\widetilde{\mathcal{O}}(\lambda \cdot \ell)\end{array}$ & \multirow[t]{2}{*}{ yes } \\
\hline & & & & & & Ver: $\widetilde{\mathcal{O}}(\lambda)$ & \\
\hline
\end{tabular}

Table 1. Comparison of known lattice-based group signatures, in terms of efficiency and functionality. The comparison is done based on two governing parameters: security parameter $\lambda$ and the maximum expected number of group users $N=2^{\ell}$. As for the scheme from [27], $R$ denotes the number of revoked users at the epoch in question. In the last column, " $\star$ means that the respective scheme can directly achieve the deniability feature via our technique, while "?" indicates that we do not know how to do so for the respective scheme.

For a security parameter parameter $\lambda$ and maximum expected number of group users $N$, our scheme features signature size $\widetilde{\mathcal{O}}(\lambda \cdot \log N)$ and group public key size $\widetilde{\mathcal{O}}\left(\lambda^{2}+\lambda \cdot \log N\right)$. The user's secret key has bit-size $\widetilde{\mathcal{O}}(\lambda)+\log N$. At each epoch when the group information is updated, the verifiers only need to 
download an extra $\widetilde{\mathcal{O}}(\lambda)$ bits in order to perform verification of signatures, 2 , while each active signer only has to download $\widetilde{\mathcal{O}}(\lambda \cdot \log N)$ bits. In Table 1, we give a detailed comparison of our scheme with known lattice-based group signatures, in terms of efficiency and functionality. The full dynamicity feature is achieved with a very reasonable cost and without having to rely on lattice trapdoors. Somewhat surprisingly, our scheme even produces shorter signatures than the scheme from [30] - which is arguably the most efficient lattice-based group signature known to date. Furthermore, these results are obtained in a relatively simple manner, thanks to three main ideas/techniques discussed below.

Our starting point is the scheme [30, which works in the static setting. Instead of relying on trapdoor-based ordinary signatures as in prior works, the LLNW scheme employs on a SIS-based Merkle tree accumulator. For a group of $N=2^{\ell}$ users, the manager chooses uniformly random vectors $\mathbf{x}_{0}, \ldots, \mathbf{x}_{N-1}$; hashes them to $\mathbf{p}_{0}, \ldots, \mathbf{p}_{N-1}$, respectively; builds a tree on top of these hash values; and publishes the tree root $\mathbf{u}$. The signing key of user $i$ consists of $\mathbf{x}_{i}$ and the witness for the fact that $\mathbf{p}_{i}$ was accumulated in $\mathbf{u}$. When issuing signatures, the user proves knowledge of a valid pair $\left(\mathbf{x}_{i}, \mathbf{p}_{i}\right)$ and of the tree path from $\mathbf{p}_{i}$ to $\mathbf{u}$. The user also has to encrypt the binary representation $\operatorname{bin}(i)$ of his identity $i$, and prove that the ciphertext is well-formed. The encryption layer is also lattice-trapdoor-free, since it utilizes the Naor-Yung double-encryption paradigm [41] with Regev's LWE-based encryption scheme [45]. To upgrade the LLNW scheme directly into a fully dynamic group signature, we now let the user compute the pair $\left(\mathbf{x}_{i}, \mathbf{p}_{i}\right)$ on his own (for enabling non-frameability), and we employ the following three ideas/techniques.

First, we add a dynamic ingredient into the static Merkle tree accumulator from [30. To this end, we equip it with an efficient updating algorithm with complexity $\mathcal{O}(\log N)$ : to change an accumulated value, we simply update the values at the corresponding leaf and along its path to the root.

Second, we create a simple rule to handle user enrollment and revocation efficiently (i.e., without resetting the whole tree). Specifically, we use the updating algorithm to set up the system so that: (i)- If a user has not joined the group or has been revoked, the value at the leaf associated with him is set as $\mathbf{0}$; (ii)- When a user joins the group, that value is set as his public key $\mathbf{p}_{i}$. Our setup guarantees that only active users (i.e., who has joined and has not been revoked at the given epoch) have their non-zero public keys accumulated into the updated root. This rule effectively separates active users who can sign from those who cannot: when signing messages, the user proceeds as in the LLNW scheme, and is asked to additionally prove in zero-knowledge that $\mathbf{p}_{i} \neq \mathbf{0}$. In other words, the seemingly big gap between being fully static and being fully dynamic has been reduced to a small difference!

\footnotetext{
${ }^{2}$ We remark that in the DDH-based instantiation from [7] which relies on the accountable ring signature from [8, the verifiers have to periodically download public keys of active signers. Our scheme overcomes this issue, thanks to the use of an updatable accumulator constructed in Section 3 .
} 
Third, the arising question now is how to additionally prove the inequality $\mathbf{p}_{i} \neq \mathbf{0}$ in the framework of the Stern-like [49] protocol from [30. One would naturally expect that this extra job could be done without losing too much in terms of efficiency. Here, the surprising and somewhat unexpected fact is that we can actually do it while gaining efficiency, thanks to the following simple idea. Recall that, in [30, to prove knowledge of $\mathbf{p}_{i} \in\{0,1\}^{n k}$, an extension technique from [35] is employed, in which $\mathbf{p}_{i}$ is extended into a vector of dimension $2 n k$. We note that, the authors of [35] also suggested a slightly modified version of their technique, that allows to simultaneously prove that $\mathbf{p}_{i} \in\{0,1\}^{n k}$ and $\mathbf{p}_{i}$ is nonzero while working only with dimension $2 n k-1$. This intriguing tweak enables us to obtain a zero-knowledge protocol with slightly lower communication cost, and hence, group signatures with slightly smaller size than in [30.

Another problem we have to overcome is that the fully dynamic setting requires a proof of correct opening, which boils down to proving correct decryption for Regev's encryption scheme [45. It involves modular linear equations with bounded-norm secrets, and can be handled using Stern-like techniques from 35128 .

Now, to equip the obtained fully dynamic group signature scheme with the deniability functionality, we first incorporate Ishida et al.'s notion of deniability [22] into Bootle et al.'s model [7]. Then we describe how to make our scheme deniable, via a zero-knowledge argument of knowledge that a given Regev ciphertext [45] does not decrypt to a given message. The latter reduces to proving knowledge of a non-zero vector $\mathbf{b} \in\{-1,0,1\}^{\ell}$ satisfying a modular linear equation, where $\mathbf{b}$ is the difference between the result of decryption and the given message. This in turn can be handled by developing the above method for proving inequality in the framework of Stern's protocol.

To summarize, we solve the prominent open question of achieving full dynamicity in the field of lattice-based group signatures. The scheme can be easily extended to a deniable group signature. Moreover, our solution is simple and comparatively efficient. Our results, while not yielding truly practical schemes, would certainly help to enrich the field and bring lattice-based group signatures one step closer to practice.

Remarks. This article is the full, extended version of [37, published in the proceedings of ACNS 2017. In comparison with [37, the present version additionally introduces the deniability feature to the obtained fully dynamic group signature scheme, and provides details of all the security proofs.

Organization. In Section 2, we recall some background on fully dynamic group signatures and lattice-based cryptography. Section 3 develops an updatable Merkle tree accumulator. Our main scheme is constructed and analyzed in Section 4. In Section 5, we demonstrate how to achieve deniability. Some details are deferred to the appendix. 


\section{Preliminaries}

\subsection{Notations}

In this paper, all vectors are column vectors. The concatenation two column vectors $\mathbf{x} \in \mathbb{R}^{m}$ and $\mathbf{y} \in \mathbb{R}^{k}$ is denoted by $(\mathbf{x} \| \mathbf{y}) \in \mathbb{R}^{m+k}$ for simplicity. Let $\|\cdot\|$ and $\|\cdot\|_{\infty}$ be the Euclidean $\left(\ell_{2}\right)$ norm and infinity $\left(\ell_{\infty}\right)$ norm of a vector respectively. For $a \in \mathbb{R}, \log a$ denotes logarithm of $a$ with base 2. Denote $[m]$ as the set $\{1,2, \cdots, m\}$.

\subsection{Fully Dynamic Group Signatures}

We recall the definition and security notions of fully dynamic group signatures (FDGS) presented by Bootle et al. 7]. An FDGS scheme involves the following entities: a group manager GM that determines who can join the group, a tracing manager TM who can open signatures, and a set of users who are potential group members. Users can join/leave the group at the discretion of GM. We assume GM will publish some information info $\tau$ regularly associated with a distinct index $\tau$ (referred as epoch hereafter). Without loss of generality, assume there is one-to-one correspondence between information and the associated epoch. The information describes changes of the group, e.g., current group members or members that are excluded from the group. We assume the published group information is authentic. By comparing current group information with previous one, it allows any party to identify revoked users at current epoch. For simplicity assume $\tau_{1}<\tau_{2}$ if info $_{\tau_{1}}$ is published before info $\tau_{2}$, i.e., the epoch preserves the order in which the corresponding group information was published. In existing models, the keys of authorities are supposed to be generated honestly; while in [7, Bootle et al. consider a stronger model where the keys of authorities can be maliciously generated by the adversary.

Syntax of fully dynamic group signatures. An FDGS scheme is a tuple of following polynomial-time algorithms.

$\operatorname{GSetup}(\lambda) \rightarrow$ pp. On input security parameter $\lambda$, this algorithm generates public parameters $\mathrm{pp}$.

$\left\langle\operatorname{GKgen}_{\mathrm{GM}}(\mathrm{pp}), \mathrm{GKgen}_{\mathrm{TM}}(\mathrm{pp})\right\rangle$. This is an interactive protocol between the group manager GM and the tracing manager TM. If it completes successfully, al-

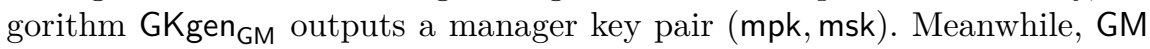
initializes the group information info and the registration table reg. The algorithm GKgen $_{\mathrm{TM}}$ outputs a tracing key pair (tpk, tsk). Set group public key $\mathrm{gpk}=(\mathrm{pp}, \mathrm{mpk}, \mathrm{tpk})$.

UKgen $(p p) \rightarrow($ upk, usk). Given public parameters pp, this algorithm generates a user key pair (upk, usk).

$\left\langle\right.$ Join( info $_{\tau_{\text {current }}}$, gpk, upk, usk); Issue $\left(\right.$ info $_{\tau_{\text {current }}}$, msk, upk $\left.)\right\rangle$. This is an interactive algorithm run by a user and GM. If it completes successfully, this user becomes a group member with an identifier uid and the Join algorithm stores 
secret group signing key gsk[uid] while Issue algorithm stores registration information in the table reg with index uid.

GUpdate(gpk, msk, info $\left.\tau_{\tau_{\text {current }}}, \mathcal{S}, \mathbf{r e g}\right) \rightarrow$ info $_{\tau_{\text {new }}}$. This is an algorithm run by GM to update group information while advancing the epoch. Given gpk,msk, info $_{\tau_{\text {current }}}$, registration table reg, a set $\mathcal{S}$ of active users to be removed from the group, GM computes new group information info ${ }_{\tau_{\text {new }}}$ and may update the table reg. If there is no change to the group, GM outputs $\perp$.

$\operatorname{Sign}\left(\right.$ gpk, gsk[uid], $\left.\operatorname{info}_{\tau}, M\right) \rightarrow \Sigma$. This algorithm outputs a group signature $\Sigma$ on message $M$ by user uid. It outputs $\perp$ if this user is inactive at epoch $\tau$.

Verify $\left(\right.$ gpk info $\left._{\tau}, M, \Sigma\right) \rightarrow 0 / 1$. This algorithm checks the validity of the signature $\Sigma$ on message $M$ at epoch $\tau$.

Trace (gpk, tsk, info $_{\tau}$, reg, $\left.M, \Sigma\right) \rightarrow$ (uid, $\Pi_{\text {trace }}$ ). This is an algorithm run by TM. Given the inputs, TM returns an identity uid of a group member who signed the message and a proof indicating this tracing result or $\perp$ if it fails to trace to a group member.

Judge(gpk, uid, info $\left.\tau, \Pi_{\text {trace }}, M, \Sigma\right) \rightarrow 0 / 1$. This algorithm checks the validity of $\Pi_{\text {trace }}$ outputted by the Trace algorithm.

Correctness and security of fully dynamic group signatures. As put forward by Bootle et al. [7, an FDGS scheme must satisfy correctness, anonymity, non-frameability, traceability and tracing soundness.

Correctness demands that a signature generated by an honest and active user is always accepted by algorithm Verify, and that algorithm Trace can always identify that user, as well as produces a proof accepted by algorithm Judge.

Anonymity requires that it is infeasible for any PPT adversary to distinguish signatures generated by two active users of its choice at the chosen epoch, even if it can corrupt any user, can choose the key of GM, and is given access to the Trace oracle.

Non-Frameability makes sure that the adversary cannot generate a valid signature that traces to an honest user even if it can corrupt all other users, and can choose keys of both managers.

Traceability ensures that the adversary cannot produce a valid signature that cannot be traced to an active user at the chosen epoch, even if it can corrupt any user and can choose the key of TM.

Tracing Soundness requires that it is infeasible to produce a valid signature that traces to two different users, even if all group users and both managers are fully controlled by the adversary.

We will define these requirements using a set of experiments, in which the adversary has access to a set of oracles. The oracles use the following global variables: HUL is a list of users whose keys are generated honestly. BUL is a list of users whose secret signing keys are revealed to the adversary; CUL is a list of users whose public keys are chosen by the adversary; SL is a set of signatures that are generated by the oracle Sign; $\mathrm{CL}$ is a set of signatures that are generated by the oracle $\mathrm{Chal}_{b}$. 
AddU(uid). This oracle adds an honest user uid to the group at current epoch. It returns upk[uid] and adds uid to the list HUL.

CrptU(uid, pk). This oracle allows the adversary to create a new user by choosing the key upk[uid] as pk. It then adds uid to the list CUL. It returns $\perp$ if uid $\in$ HUL $\cup$ CUL. This actually prepares for calling the oracle SndToGM.

SndToGM(uid, $M_{\text {in }}$ ). This oracle engages in the 〈Join, Issue $\rangle$ protocol between the adversary, who corrupts the user uid in the list CUL, and the honest Issue-executing group manager.

SndToU(uid, $M_{\text {in }}$ ). This oracle engages in the 〈Join, Issue $\rangle$ protocol between the adversary, who corrupts the group manager, and the honest Join-executing user uid in the list HUL.

RReg(uid). This oracle returns registration information $\mathbf{r e g}$ [uid] of user uid.

MReg(uid, $\rho$ ). This oracle modifies reg[uid] to any $\rho$ chosen by the adversary.

RevealU(uid). This oracle returns secret signing key gsk[uid] to the adversary, and adds this user to the list BUL.

Sign(uid, $M, \tau$ ). This oracle returns a signature on the message $M$ by the user uid at epoch $\tau$, and then add the signature to the list SL.

$\mathrm{Chal}_{b}\left(\mathrm{info}_{\tau}, \mathrm{uid}_{0}, \mathrm{uid}_{1}, M\right)$. This oracle receives inputs from the adversary, returns a signature on $M$ by the user uid $b$ at epoch $\tau$, and then adds the signature to the list CL. It is required that the two challenged users are active at epoch $\tau$ and this oracle can only be called once.

$\operatorname{Trace}\left(M, \Sigma, \operatorname{Info}_{\tau}\right)$. This oracle returns the signer of the signature together with a proof, with respect to the epoch $\tau$. We require that the signature is not in the list $\mathrm{CL}$.

$\operatorname{GUpdate}(\mathcal{S})$. This oracle allows the adversary to update the group at current epoch $\tau_{\text {current }}$. It is required that $\mathcal{S}$ is a set of active users at current epoch.

We also need the following polynomial-time algorithm in security experiments to ease composition.

IsActive $\left(\right.$ info $_{\tau}$, reg, uid $) \rightarrow 0 / 1$ : It outputs 1 if this user is active at epoch $\tau$ and 0 otherwise.

We refer the readers to [7] for detailed descriptions of the above oracles.

Definition 1. For any security parameter $\lambda$ and any PPT adversary $\mathcal{A}$, we define correctness and security experiments in Fig. 1.

For correctness, non-frameability, traceability and tracing soundness, the advantage of the adversary is defined as the probability of outputting 1 in the corresponding experiment. For anonymity, the advantage is defined as the absolute difference of probability of outputting 1 between experiment $\operatorname{Exp}_{\mathrm{FDGS}, \mathcal{A}}^{\mathrm{anon}-1}$ and experiment $\mathbf{E x p}_{\mathrm{FDGS}, \mathcal{A}}^{\text {anon }}$.

A fully dynamic group signature scheme is said to be correct and secure (i.e., anonymous, non-frameable, traceable and tracing sound) if the advantages of the adversary in all considered experiments are negligible in $\lambda$. 
$\frac{\text { Experiment: } \operatorname{Exp}_{\mathrm{FDGS}, \mathcal{A}}^{\text {corr }}(\lambda)}{\mathrm{pp} \leftarrow \mathrm{GSetup}(\lambda), \mathrm{HUL}:=\emptyset .}$
$\langle($ info, mpk, msk $) ;($ tpk, tsk $)\rangle \leftarrow\langle$ GKgen
GM $\left.(\mathrm{pp}), \mathrm{GKgen}_{\mathrm{TM}}(\mathrm{pp})\right\rangle$.

Set gpk $=$ (pp, mpk, tpk). (uid, $M, \tau) \leftarrow \mathcal{A}^{\text {AddU, RReg,GUpdate }}$ (gpk, info).

If uid $\notin \mathrm{HUL}$ or gsk[uid] $=\perp$ or info $_{\tau}=\perp$ or IsActive(info ${ }_{\tau}$, reg, uid) $=0$, return 0 . $\Sigma \leftarrow \operatorname{Sign}($ gpk, gsk[uid], info $\tau, M),\left(\right.$ uid $\left.^{*}, \Pi_{\text {trace }}\right) \leftarrow$ Trace(gpk, tsk, info ${ }_{\tau}$, reg, $\left.M, \Sigma\right)$. Return 1 if (Verify $($ gpk, info $\tau, M, \Sigma)=0 \vee$ Judge (gpk, uid, info $\left.{ }_{\tau}, \Pi_{\text {trace }}, m, \Sigma\right)=$ $0 \vee$ uid $\neq$ uid $^{*}$ ).

Experiment: $\operatorname{Exp}_{\mathrm{FDGS}, \mathcal{A}}^{\mathrm{anon}-\mathrm{b}}(\lambda)$

$\overline{\mathrm{pp}} \leftarrow \mathrm{GSetup}(\lambda), \mathrm{HUL}, \mathrm{CUL}, \mathrm{BUL}, \mathrm{CL}, \mathrm{SL}:=\emptyset$.

(st init $_{\left.\text {, info, } \mathrm{mpk}, \mathrm{msk}) \leftarrow \mathcal{A}^{\langle\cdot, \mathrm{GKgen}} \mathrm{TM}\right\rangle(\mathrm{pp})}$ (init : pp).

Return 0 if GKgen $\mathrm{TM}$ did not accept or $\mathcal{A}$ 's output is not well-formed.

Denote the output of GKgen TM $_{\text {as }}$ (tpk, tsk), and set gpk $=(\mathrm{pp}, \mathrm{mpk}, \mathrm{tpk})$.

$b^{*} \leftarrow \mathcal{A}^{\text {AddU, CrptU, RevealU,SndToU,Trace,MReg,Chal }}$ (play : st init, gpk). Return $b^{*}$.

Experiment: $\operatorname{Exp}_{\mathrm{FDGS}, \mathcal{A}}^{\text {non }-\mathrm{frame}}(\lambda)$

$\mathrm{pp} \leftarrow \mathrm{GSetup}(\lambda), \mathrm{HUL}, \mathrm{CUL}, \mathrm{BUL}, \mathrm{SL}=\emptyset$.

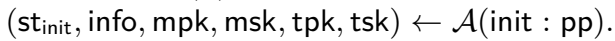

Return 0 if $\mathcal{A}$ 's output is not well-formed, otherwise set gpk $=(\mathrm{pp}, \mathrm{mpk}$, tpk).

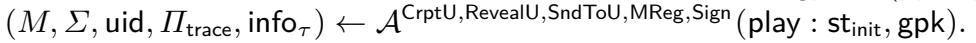

Return 1 if (Verify $\left(\right.$ gpk, $\left.^{\text {info }} \tau, M, \Sigma\right)=1 \wedge$ Judge(gpk, uid, info $\left.{ }_{\tau}, \Pi_{\text {trace }}, M, \Sigma\right)=1 \wedge$ uid $\in \mathrm{HUL} \backslash \mathrm{BUL} \wedge(M, \Sigma, \tau) \notin \mathrm{SL})$.

Experiment: $\operatorname{Exp}_{\mathrm{FDGS}, \mathcal{A}}^{\text {trace }}(\lambda)$

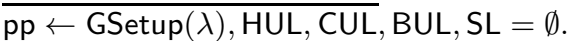

$\left(\right.$ st $_{\text {init }}$, tpk, tsk $) \leftarrow \mathcal{A}^{\left\langle\mathrm{GKgen}_{\mathrm{GM}}(\mathrm{pp}), \cdot\right\rangle}($ init : pp).

Return 0 if GKgen $_{\mathrm{GM}}$ did not accept or $\mathcal{A}$ 's output is not well-formed.

Denote the output of GKgen ${ }_{\mathrm{GM}}$ as (mpk, msk, info), and set gpk = (pp, mpk, tpk).

$(M, \Sigma, \tau) \leftarrow \mathcal{A}^{\text {AddU,CrptU,SndToGM, RevealU,MReg,Sign,GUpdate }}$ (play : $\mathrm{st}_{\text {init }}$, gpk, info).

If Verify $($ gpk, info $\tau, M, \Sigma)=0$, return 0 .

(uid, $\left.\Pi_{\text {trace }}\right) \leftarrow$ Trace $($ gpk, tsk, info $\tau$, reg, $M, \Sigma)$.

Return 1 if (IsActive $\left(\right.$ info $_{\tau}$, reg, uid) $=\perp \vee$ Judge(gpk, uid, info $\left.{ }_{\tau}, \Pi_{\text {trace }}, M, \Sigma\right)=$ $0 \vee$ uid $=0)$.

Experiment: $\operatorname{Exp}_{\mathrm{FDGS}, \mathcal{A}}^{\text {trace-sound }}(\lambda)$

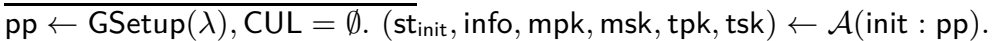

Return 0 if $\mathcal{A}$ 's output is not well-formed, otherwise set gpk $=$ (pp, mpk, tpk).

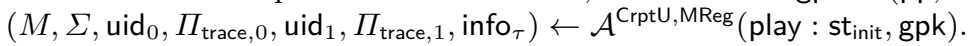

Return 1 if (Verify $\left(\right.$ gpk, info $\left._{\tau}, M, \Sigma\right)=1 \wedge \operatorname{uid}_{0}(\neq \perp) \neq \operatorname{uid}_{1}(\neq \perp) \wedge$ Judge(gpk, uid $b$, info $\left._{\tau}, \Pi_{\text {trace }, b}, M, \Sigma\right)=1$ for $\left.b \in\{0,1\}\right)$.

Fig. 1: Experiments to define security requirements of FDGS. 


\subsection{Background on Lattices}

We recall the average-case lattice problems SIS and LWE, together with their hardness results.

Definition 2 ([1] 19]). The $\mathrm{SIS}_{n, m, q, \beta}^{\infty}$ problem is as follows: Given uniformly random matrix $\mathbf{A} \in \mathbb{Z}_{q}^{n \times m}$, find a non-zero vector $\mathbf{x} \in \mathbb{Z}^{m}$ such that $\|\mathbf{x}\|_{\infty} \leq \beta$ and $\mathbf{A} \cdot \mathbf{x}=\mathbf{0} \bmod q$.

If $m, \beta=\operatorname{poly}(n)$, and $q>\beta \cdot \widetilde{\mathcal{O}}(\sqrt{n})$, then the $\operatorname{SIS}_{n, m, q, \beta}^{\infty}$ problem is at least as hard as worst-case lattice problem $\operatorname{SIVP}_{\gamma}$ for some $\gamma=\beta \cdot \widetilde{\mathcal{O}}(\sqrt{n m})$ (see, e.g., [19|39]). Specifically, when $\beta=1, q=\widetilde{\mathcal{O}}(n), m=2 n\lceil\log q\rceil$, the $\operatorname{SIS}_{n, m, q, 1}^{\infty}$ problem is at least as hard as $\operatorname{SIVP}_{\gamma}$ with $\gamma=\widetilde{\mathcal{O}}(n)$.

In the last decade, numerous SIS-based cryptographic primitives have been proposed. In this work, we will extensively employ 2 such constructions:

- Our group signature scheme is based on the Merkle tree accumulator from [30], which is built upon a specific family of collision-resistant hash functions.

- Our zero-knowledge argument systems use the statistically hiding and computationally binding string commitment scheme from 24 .

For appropriate setting of parameters, the security of the above two constructions can be based on the worst-case hardness of $\operatorname{SIVP}_{\widetilde{\mathcal{O}}(n)}$.

In the group signature in Section 4 , we will employ the multi-bit version of Regev's encryption scheme [45, presented in [23]. The scheme is based on the hardness of the LWE problem.

Definition 3 ([45]). Let $n, m \geq 1, q \geq 2$, and let $\chi$ be a probability distribution on $\mathbb{Z}$. For $\mathbf{s} \in \mathbb{Z}_{q}^{n}$, let $\mathcal{A}_{\mathbf{s}, \chi}$ be the distribution obtained by sampling $\mathbf{a} \stackrel{\$}{\leftarrow} \mathbb{Z}_{q}^{n}$ and $e \hookleftarrow \chi$, and outputting $\left(\mathbf{a}, \mathbf{s}^{\top} \cdot \mathbf{a}+e\right) \in \mathbb{Z}_{q}^{n} \times \mathbb{Z}_{q}$. The $\operatorname{LWE}_{n, q, \chi}$ problem asks to distinguish $m$ samples chosen according to $\mathcal{A}_{\mathbf{s}, \chi}$ (for $\mathbf{s} \stackrel{\$}{\leftarrow} \mathbb{Z}_{q}^{n}$ ) and $m$ samples chosen according to the uniform distribution over $\mathbb{Z}_{q}^{n} \times \mathbb{Z}_{q}$.

If $q$ is a prime power, $\chi$ is the discrete Gaussian distribution $D_{\mathbb{Z}, \alpha q}$, where $\alpha q \geq$ $2 \sqrt{n}$, then $\operatorname{LWE}_{n, q, \chi}$ is as least as hard as $\operatorname{SIVP}_{\widetilde{\mathcal{O}}(n / \alpha)}($ see [45/38/39]).

\subsection{Stern-like Protocols for Lattice-Based Relations}

The zero-knowledge (ZK) argument systems appearing in this paper operate in the framework of Stern's protocol [49. Let us now recall some background. This protocol was originally proposed in the context of code-based cryptography, and was later adapted into the lattice setting by Kawachi et al. [24]. Subsequently, it was empowered by Ling et al. [35] to handle the matrix-vector relations associated with the SIS and LWE problems, and further developed to design several lattice-based schemes: group signatures [27/36/30/31/28, policybased signatures [16] and group encryption [29]. 
Stern-like protocols are quite useful in the context of lattice-based privacypreserving systems, when one typically works with modular linear equations of the form $\sum_{i} \mathbf{M}_{i} \cdot \mathbf{x}_{i}=\mathbf{v} \bmod q-$ where $\left\{\mathbf{M}_{i}\right\}_{i}, \mathbf{v}$ are public, and one wants to prove in ZK that secret vectors $\left\{\mathbf{x}_{i}\right\}_{i}$ satisfy certain constraints, e.g., they have small norms and/or have coordinates arranged in a special way. The high-level ideas can be summarized as follows. If the given constraints are invariant under certain type of permutations of coordinates, then one readily uses uniformly random permutations to prove those constraints. Otherwise, one performs some pre-processings with $\left\{\mathbf{x}_{i}\right\}_{i}$ to reduce to the former case. Meanwhile, to prove that the modular linear equation holds, one makes use of a standard masking technique.

The basic protocol consists of 3 moves: commitment, challenge, response. If the statistically hiding and computationally binding string commitment scheme from [24] is employed in the first move, then one obtains a statistical zeroknowledge argument of knowledge (ZKAoK) with perfect completeness, constant soundness error $2 / 3$, and communication $\operatorname{cost} \mathcal{O}(|w| \cdot \log q)$, where $|w|$ denotes the total bit-size of the secret vectors. In many applications, the protocol is repeated $\kappa=\omega(\log \lambda)$ times, for security parameter $\lambda$, to achieve negligible soundness error, and then made non-interactive via the Fiat-Shamir heuristic [18. In the random oracle model, this results in a non-interactive zero-knowledge argument of knowledge (NIZKAoK) with bit-size $\mathcal{O}(|w| \cdot \log q) \cdot \omega(\log \lambda)$.

\section{Updatable Lattice-Based Merkle Hash Trees}

We first recall the lattice-based Merkle-tree accumulator from [30, and then, we equip it with a simple updating algorithm which allows to change an accumulated value in time logarithmic in the size of the accumulated set. This updatable hash tree will serve as the building block of our construction in Section 4 .

\subsection{Cryptographic Accumulators}

An accumulator scheme is a tuple of polynomial-time algorithms defined below.

$\operatorname{TSetup}(\lambda)$ On input security parameter $\lambda$, output the public parameter pp.

TAccpp On input a set $R=\left\{\mathbf{d}_{0}, \ldots, \mathbf{d}_{N-1}\right\}$ of $N$ data values, output an accumulator value $\mathbf{u}$.

TWitness $_{\text {pp }}$ On input a data set $R$ and a value $\mathbf{d}$, output $\perp$ if $\mathbf{d} \notin R$; otherwise output a witness $w$ for the fact that $\mathbf{d}$ is accumulated in $\operatorname{TAcc}(R)$. (Typically, the size of $w$ should be short (e.g., constant or logarithmic in $N$ ) to be useful.)

TVerify $_{\mathrm{pp}}$ On input accumulator value $\mathbf{u}$ and a value-witness pair $(\mathbf{d}, w)$, output 1 (which indicates that $(\mathbf{d}, w)$ is valid for the accumulator $\mathbf{u}$ ) or 0 .

An accumulator scheme is called correct if for all pp $\leftarrow \operatorname{TSetup}(\lambda)$, we have $\operatorname{TVerify}_{\mathrm{pp}}\left(\operatorname{TAcc}_{\mathrm{pp}}(R), \mathbf{d}, \operatorname{TWitness}_{\mathrm{pp}}(R, \mathbf{d})\right)=1$ for all $\mathbf{d} \in R$.

A natural security requirement for accumulators, as considered in [312 30, says that it is infeasible to prove that a value $\mathbf{d}^{*}$ was accumulated in a value $\mathbf{u}$ if it was not. This property is formalized as follows. 
Definition 4 ([30]). An accumulator scheme (TSetup, TAcc, TWitness, TVerify) is called secure if for all PPT adversaries $\mathcal{A}$ :

$$
\begin{aligned}
\operatorname{Pr}[\mathrm{pp} & \leftarrow \operatorname{TSetup}(\lambda) ;\left(R, \mathbf{d}^{*}, w^{*}\right) \leftarrow \mathcal{A}(\mathrm{pp}): \\
\mathbf{d}^{*} & \left.\notin R \wedge \operatorname{TVerify} \mathrm{pp}_{\mathrm{pp}}\left(\operatorname{TAcc}_{\mathrm{pp}}(R), \mathbf{d}^{*}, w^{*}\right)=1\right]=\operatorname{negl}(\lambda) .
\end{aligned}
$$

\subsection{The LLNW Merkle-tree Accumulator}

Notations. Hereunder we will use the notation $x \stackrel{\$}{\leftarrow}$ to indicate that $x$ is chosen uniformly at random from finite set $S$. For bit $b \in\{0,1\}$, we let $\bar{b}=1-b$. The Merkle-tree accumulator scheme from [30] works with parameters $n=\mathcal{O}(\lambda)$, $q=\widetilde{\mathcal{O}}\left(n^{1.5}\right), k=\left\lceil\log _{2} q\right\rceil$, and $m=2 n k$. The set $\mathbb{Z}_{q}$ is identified by $\{0, \ldots, q-1\}$. Define the "powers-of-2" matrix

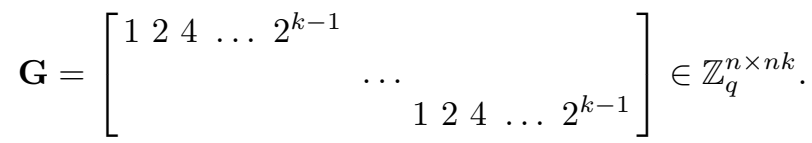

Note that for every $\mathbf{v} \in \mathbb{Z}_{q}^{n}$, we have $\mathbf{v}=\mathbf{G} \cdot \operatorname{bin}(\mathbf{v})$, where $\operatorname{bin}(\mathbf{v}) \in\{0,1\}^{n k}$ denotes the binary representation of $\mathbf{v}$. The scheme is built upon the following family of SIS-based collision-resistant hash functions.

Definition 5. The function family $\mathcal{H}$ mapping $\{0,1\}^{n k} \times\{0,1\}^{n k}$ to $\{0,1\}^{n k}$ is defined as $\mathcal{H}=\left\{h_{\mathbf{A}} \mid \mathbf{A} \in \mathbb{Z}_{q}^{n \times m}\right\}$, where for $\mathbf{A}=\left[\mathbf{A}_{0} \mid \mathbf{A}_{1}\right]$ with $\mathbf{A}_{0}, \mathbf{A}_{1} \in \mathbb{Z}_{q}^{n \times n k}$, and for any $\left(\mathbf{u}_{0}, \mathbf{u}_{1}\right) \in\{0,1\}^{n k} \times\{0,1\}^{n k}$, we have:

$$
h_{\mathbf{A}}\left(\mathbf{u}_{0}, \mathbf{u}_{1}\right)=\operatorname{bin}\left(\mathbf{A}_{0} \cdot \mathbf{u}_{0}+\mathbf{A}_{1} \cdot \mathbf{u}_{1} \bmod q\right) \in\{0,1\}^{n k} .
$$

Note that $h_{\mathbf{A}}\left(\mathbf{u}_{0}, \mathbf{u}_{1}\right)=\mathbf{u} \Leftrightarrow \mathbf{A}_{0} \cdot \mathbf{u}_{0}+\mathbf{A}_{1} \cdot \mathbf{u}_{1}=\mathbf{G} \cdot \mathbf{u} \bmod q$.

A Merkle tree with $N=2^{\ell}$ leaves, where $\ell$ is a positive integer, then can be constructed based on the function family $\mathcal{H}$ as follows.

$\operatorname{TSetup}(\lambda)$. Sample $\mathbf{A} \stackrel{\$}{\leftarrow} \mathbb{Z}_{q}^{n \times m}$, and output $\mathrm{pp}=\mathbf{A}$.

$\operatorname{TAcc}_{\mathbf{A}}\left(R=\left\{\mathbf{d}_{0} \in\{0,1\}^{n k}, \ldots, \mathbf{d}_{N-1} \in\{0,1\}^{n k}\right\}\right)$. For every $j \in[0, N-1]$, let $\operatorname{bin}(j)=\left(j_{1}, \ldots, j_{\ell}\right) \in\{0,1\}^{\ell}$ be the binary representation of $j$, and let $\mathbf{d}_{j}=\mathbf{u}_{j_{1}, \ldots, j_{\ell}}$. Form the tree of depth $\ell=\log N$ based on the $N$ leaves $\mathbf{u}_{0,0, \ldots, 0}, \ldots, \mathbf{u}_{1,1, \ldots, 1}$ as follows:

1. At depth $i \in[\ell]$, the node $\mathbf{u}_{b_{1}, \ldots, b_{i}} \in\{0,1\}^{n k}$, for all $\left(b_{1}, \ldots, b_{i}\right) \in\{0,1\}^{i}$, is defined as $h_{\mathbf{A}}\left(\mathbf{u}_{b_{1}, \ldots, b_{i}, 0}, \mathbf{u}_{b_{1}, \ldots, b_{i}, 1}\right)$.

2. At depth 0: The root $\mathbf{u} \in\{0,1\}^{n k}$ is defined as $h_{\mathbf{A}}\left(\mathbf{u}_{0}, \mathbf{u}_{1}\right)$.

The algorithm outputs the accumulator value $\mathbf{u}$.

$\operatorname{TWitness}_{\mathbf{A}}(R, \mathbf{d})$. If $\mathbf{d} \notin R$, return $\perp$. Otherwise, $\mathbf{d}=\mathbf{d}_{j}$ for some $j \in[0, N-1]$ with binary representation $\left(j_{1}, \ldots, j_{\ell}\right)$. Output the witness $w$ defined as:

$$
w=\left(\left(j_{1}, \ldots, j_{\ell}\right),\left(\mathbf{u}_{j_{1}, \ldots, j_{\ell-1}, \overline{j_{\ell}}}, \ldots, \mathbf{u}_{j_{1}, \overline{j_{2}}}, \mathbf{u}_{\overline{j_{1}}}\right)\right) \in\{0,1\}^{\ell} \times\left(\{0,1\}^{n k}\right)^{\ell},
$$

for $\mathbf{u}_{j_{1}, \ldots, j_{\ell-1}, \overline{j_{\ell}}}, \ldots, \mathbf{u}_{j_{1}, \overline{j_{2}}}, \mathbf{u}_{\overline{j_{1}}}$ computed by algorithm $\operatorname{TAcc}_{\mathbf{A}}(R)$. 
$\operatorname{TVerify}_{\mathbf{A}}(\mathbf{u}, \mathbf{d}, w)$. Let the given witness $w$ be of the form:

$$
w=\left(\left(j_{1}, \ldots, j_{\ell}\right),\left(\mathbf{w}_{\ell}, \ldots, \mathbf{w}_{1}\right)\right) \in\{0,1\}^{\ell} \times\left(\{0,1\}^{n k}\right)^{\ell} .
$$

The algorithm recursively computes the path $\mathbf{v}_{\ell}, \mathbf{v}_{\ell-1}, \ldots, \mathbf{v}_{1}, \mathbf{v}_{0} \in\{0,1\}^{n k}$ as follows: $\mathbf{v}_{\ell}=\mathbf{d}$ and

$$
\forall i \in\{\ell-1, \ldots, 1,0\}: \mathbf{v}_{i}=\left\{\begin{array}{l}
h_{\mathbf{A}}\left(\mathbf{v}_{i+1}, \mathbf{w}_{i+1}\right), \text { if } j_{i+1}=0 ; \\
h_{\mathbf{A}}\left(\mathbf{w}_{i+1}, \mathbf{v}_{i+1}\right), \text { if } j_{i+1}=1 .
\end{array}\right.
$$

Then it returns 1 if $\mathbf{v}_{0}=\mathbf{u}$. Otherwise, it returns 0 .

The following lemma states the correctness and security of the above Merkle tree accumulator.

Lemma 1 ([30]). The given accumulator scheme is correct and is secure in the sense of Definition 4, assuming the hardness of the $\mathrm{SIS}_{n, m, q, 1}^{\infty}$ problem.

\subsection{An Efficient Updating Algorithm}

Unlike the static group signature scheme from [30], our fully dynamic construction of Section 4 requires to regularly edit the accumulated values without having to reconstruct the whole tree. To this end, we equip the Merkle tree accumulator from [30. with a simple, yet efficient, updating algorithm: to change the value at a given leaf, we simply modify all values in the path from that leaf to the root. The algorithm, which takes as input a bit string $\operatorname{bin}(j)=\left(j_{1}, j_{2}, \ldots, j_{\ell}\right)$ and a value $\mathbf{d}^{*} \in\{0,1\}^{n k}$, is formally described below.

Given the tree in Section 3.2. algorithm TUpdate $\mathbf{A}\left(\left(j_{1}, j_{2}, \ldots, j_{\ell}\right), \mathbf{d}^{*}\right)$ performs the following steps:

1. Let $\mathbf{d}_{j}$ be the current value at the leaf of position determined by $\operatorname{bin}(j)$, and let $\left(\left(j_{1}, \ldots, j_{\ell}\right),\left(\mathbf{w}_{j, \ell}, \ldots, \mathbf{w}_{j, 1}\right)\right)$ be its associated witness.

2. Set $\mathbf{v}_{\ell}:=\mathbf{d}^{*}$ and recursively compute the path $\mathbf{v}_{\ell}, \mathbf{v}_{\ell-1}, \ldots, \mathbf{v}_{1}, \mathbf{v}_{0} \in\{0,1\}^{n k}$ as in (11).

3. Set $\mathbf{u}:=\mathbf{v}_{0} ; \mathbf{u}_{j_{1}}:=\mathbf{v}_{1} ; \ldots ; \mathbf{u}_{j_{1}, j_{2}, \ldots, j_{\ell-1}}:=\mathbf{v}_{\ell-1} ; \mathbf{u}_{j_{1}, j_{2}, \ldots, j_{\ell}}:=\mathbf{v}_{\ell}=\mathbf{d}^{*}$.

It can be seen that the provided algorithm runs in time $\mathcal{O}(\ell)=\mathcal{O}(\log N)$. In Fig. 2, we give an illustrative example of a tree with $2^{3}=8$ leaves.

\section{Our Fully Dynamic Group Signatures from Lattices}

In this section, we construct our lattice-based fully dynamic group signature and prove its security in Bootle et al's model [7]. We start with the LLNW scheme [30, which works in the static setting.

While other constructions of lattice-based group signatures employ trapdoorbased ordinary signature schemes (e.g., 914]) to certify users, the LLNW scheme relies on a SIS-based Merkle tree accumulator which we recalled in Section 3.2. 


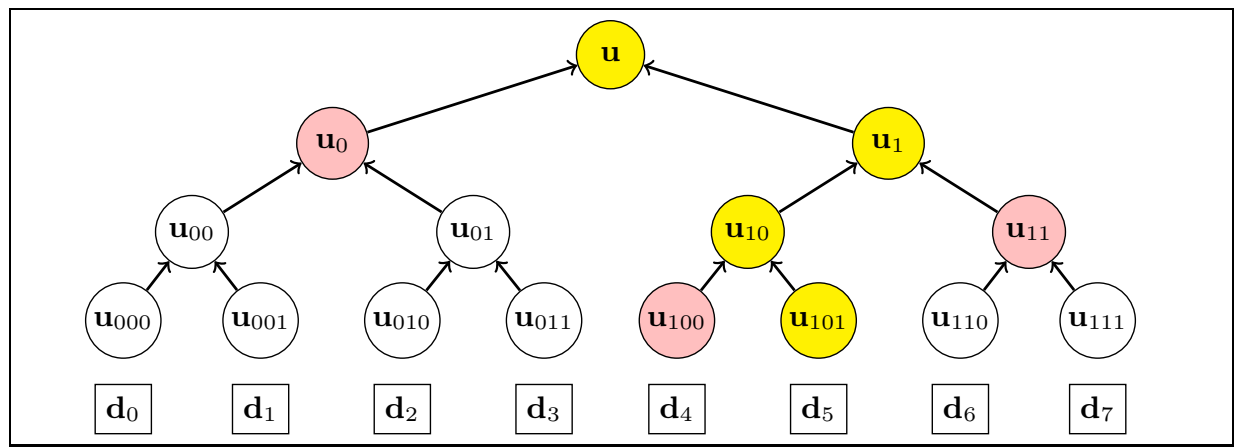

Fig. 2: A Merkle tree with $2^{3}=8$ leaves, which accumulates the data blocks $\mathbf{d}_{0}, \ldots, \mathbf{d}_{7}$ into the value $\mathbf{u}$ at the root. The bit string (101) and the pink nodes form a witness to the fact that $\mathbf{d}_{5}$ is accumulated in $\mathbf{u}$. If we replace $\mathbf{d}_{5}$ by a new value $\mathbf{d}^{*}$, we only need to update the yellow nodes.

The GM, who manages a group of $N=2^{\ell}$ users, chooses uniformly random vectors $\mathbf{x}_{0}, \ldots, \mathbf{x}_{N-1} \in\{0,1\}^{m}$; hashes them to $\mathbf{p}_{0}, \ldots, \mathbf{p}_{N-1} \in\{0,1\}^{n k}$, respectively; builds a tree on top of these hash values; and lets the tree root $\mathbf{u} \in\{0,1\}^{n k}$ be part of the group public key. The signing key of user $i$ consists of $\mathbf{x}_{i}$ and the witness for the fact that $\mathbf{p}_{i}$ was accumulated in $\mathbf{u}$. When generating group signatures, the user proves knowledge of a valid pair $\left(\mathbf{x}_{i}, \mathbf{p}_{i}\right)$ and of the tree path from $\mathbf{p}_{i}$ to $\mathbf{u}$. The user also has to encrypt the binary representation $\operatorname{bin}(i)$ of his identity $i$, and prove that the ciphertext is well-formed. The encryption layer utilizes the Naor-Yung double-encryption paradigm [41] with Regev's LWE-based cryptosystem, and thus, it is also lattice-trapdoor-free.

To upgrade the LLNW scheme directly into a fully dynamic group signature, some tweaks and new ideas are needed. First, to enable the non-frameability feature, we let the user compute the pair $\left(\mathbf{x}_{i}, \mathbf{p}_{i}\right)$ on his own. The second problem we have to consider is that Merkle hash trees seem to be a static primitive. To this end, we equip the accumulator with an efficient updating algorithm (see Section 3.3). Now, the challenging question is how to handle user enrollment and revocation in a simple manner (i.e., without having to reset the whole tree). To tackle these issues, we associate each of the $N$ potential users with a leaf in the tree, and then use the updating algorithm to set up the system so that:

1. If a user has not joined the group or has been revoked, the value at the leaf associated with him is set as $\mathbf{0}$;

2. When a user joins the group, that value is set as his public key $\mathbf{p}_{i}$.

Our setup guarantees that only active users (i.e., who has joined and has not been revoked at the given epoch) have their non-zero public keys accumulated into the updated root. This effectively gives us a method to separate active users who can sign from those who cannot: when signing messages, the user proceeds as in the LLNW scheme, and is asked to additionally prove in ZK that $\mathbf{p}_{i} \neq \mathbf{0}$. 
At this point, the arising question is how to prove the inequality $\mathbf{p}_{i} \neq \mathbf{0}$ in the framework of the Stern-like [49] protocol from [30. One would naturally hope that this extra job could be done without losing too much in terms of efficiency. Here, the surprising and somewhat unexpected fact is that we can actually do it while gaining efficiency, thanks to a technique originally proposed in 35 .

To begin with, let $B_{t}^{L}$ denote the set of all vectors in $\{0,1\}^{L}$ having Hamming weight exactly $t$. In Stern-like protocols (see Section 2.4), a common technique for proving in ZK the possession of $\mathbf{p} \in\{0,1\}^{n k}$ consists of appending $n k$ "dummy" entries to it to obtain $\mathbf{p}^{*} \in \mathrm{B}_{n k}^{2 n k}$, and demonstrating to the verifier that a random permutation of $\mathbf{p}^{*}$ belongs to the "target set" $\mathrm{B}_{n k}^{2 n k}$. This suffices to convince the verifier that the original vector $\mathbf{p}$ belongs to $\{0,1\}^{n k}$, while the latter cannot learn any additional information about $\mathbf{p}$, thanks to the randomness of the permutation. This extending-then-permuting technique was first proposed in [35], and was extensively used in the underlying protocol of the LLNW scheme. Now, to address our question, we will employ a modified version of this technique, which was also initially suggested in 35. Let us think of another "target set", so that it is possible to extend $\mathbf{p} \in\{0,1\}^{n k}$ to an element of that set if and only if $\mathbf{p}$ is non-zero. That set is $\mathrm{B}_{n k}^{2 n k-1}$. Indeed, the extended vector $\mathbf{p}^{*}$ belongs to $\mathrm{B}_{n k}^{2 n k-1}$ if and only if the original vector has Hamming weight at least $n k-(n k-1)=1$, which means that it cannot be a zero-vector. When combining with the permuting step, this modification allows us to additionally prove the given inequality while working with smaller dimension. As a result, our fully dynamic scheme produces slightly shorter signatures than the original static scheme.

Finally, we remark that the fully dynamic setting requires a proof of correct opening, which boils down to proving correct decryption for Regev's encryption scheme. It involves modular linear equations with bounded-norm secrets, and can be easily handled using Stern-like techniques from 3528.

\subsection{Description of the Scheme}

Our scheme is described as follows.

$\operatorname{GSetup}(\lambda)$. On input security parameter $\lambda$, this algorithm specifies the following: - An expected number of potential users $N=2^{\ell}=\operatorname{poly}(\lambda)$.

- Dimension $n=\mathcal{O}(\lambda)$, prime modulus $q=\widetilde{\mathcal{O}}\left(n^{1.5}\right)$, and $k=\left\lceil\log _{2} q\right\rceil$. These parameters implicitly determine the "powers-of-2" matrix $\mathbf{G} \in$ $\mathbb{Z}_{q}^{n \times n k}$, as defined in Section 3 .

- Matrix dimensions $m=2 n k$ for the hashing layer, and $m_{E}=2(n+\ell) k$ for the encryption layer.

- An integer $\beta=\sqrt{n} \cdot \omega(\log n)$, and a $\beta$-bounded noise distribution $\chi$.

- A hash function $\mathcal{H}_{\mathrm{FS}}:\{0,1\}^{*} \rightarrow\{1,2,3\}^{\kappa}$, where $\kappa=\omega(\log \lambda)$, to be modelled as a random oracle in the Fiat-Shamir transformations [18.

- Let COM : $\{0,1\}^{*} \times\{0,1\}^{m} \rightarrow \mathbb{Z}_{q}^{n}$ be the string commitment scheme from [24, to be used in our zero-knowledge argument systems. 
- Uniformly random matrix $\mathbf{A} \in \mathbb{Z}_{q}^{n \times m}$.

The algorithm outputs public parameters

$$
\mathrm{pp}=\left\{\lambda, N, n, q, k, m, m_{E}, \ell, \beta, \chi, \kappa, \mathcal{H}_{\mathrm{FS}}, \mathrm{COM}, \mathbf{A}\right\} .
$$

$\left\langle\right.$ GKgen $_{\mathrm{GM}}(\mathrm{pp})$, GKgen $\left.\mathrm{TM}(\mathrm{pp})\right\rangle$. The group manager GM and the tracing manager TM initialize their keys and the public group information as follows.

- $\operatorname{GKgen}_{\mathrm{GM}}(\mathrm{pp})$. This algorithm samples msk $\$\{0,1\}^{m}$ and computes $\mathrm{mpk}=\mathbf{A} \cdot \operatorname{msk} \bmod q$, and outputs (mpk, msk). Here, we consider $\mathrm{mpk}$ as an identifier of the group managed by GM who has mpk as his public key. Furthermore, as in [7, Sec. 3.3, full version], we assume that the group information board is visible to everyone, but can only be edited by a party knowing msk.

- GKgen ${ }_{T M}(\mathrm{pp})$. This algorithm initializes the Naor-Yung double-encryption mechanism with the $\ell$-bit version Regev encryption scheme. It first chooses $\mathbf{B} \stackrel{\$}{\leftarrow} \mathbb{Z}_{q}^{n \times m_{E}}$. For each $i \in\{1,2\}$, it samples $\mathbf{S}_{i} \stackrel{\$}{\leftarrow} \chi^{n \times \ell}, \mathbf{E}_{i} \hookleftarrow \chi^{\ell \times m_{E}}$, and computes $\mathbf{P}_{i}=\mathbf{S}_{i}^{\top} \cdot \mathbf{B}+\mathbf{E}_{i} \in \mathbb{Z}_{q}^{\ell \times m_{E}}$. Then, TM sets tsk $=\left(\mathbf{S}_{1}, \mathbf{E}_{1}\right)$, and tpk $=\left(\mathbf{B}, \mathbf{P}_{1}, \mathbf{P}_{2}\right)$.

- TM sends tpk to GM who initializes the following:

- Table reg $:=(\operatorname{reg}[0][1], \operatorname{reg}[0][2], \ldots, \operatorname{reg}[N-1][1], \operatorname{reg}[N-1][2])$, where for each $i \in[0, N-1]: \operatorname{reg}[i][1]=0^{n k}$ and $\operatorname{reg}[i][2]=0$. Looking ahead, $\operatorname{reg}[i][1]$ will be used to record the public key of a registered user, while $\operatorname{reg}[i][2]$ stores the epoch at which the user joins.

- The Merkle tree $\mathcal{T}$ built on top of $\operatorname{reg}[0][1], \ldots, \operatorname{reg}[N-1][1]$. (Note that $\mathcal{T}$ is an all-zero tree at this stage, but it will be modified when a new user joins the group, or when GM computes the updated group information.)

- Counter of registered users $c:=0$.

Then, GM outputs gpk $=(\mathrm{pp}, \mathrm{mpk}, \mathrm{tpk})$ and announces the initial group information info $=\emptyset$. He keeps $\mathcal{T}$ and $c$ for himself.

UKgen(pp). Each potential group user samples usk $=\mathbf{x} \stackrel{\$}{\leftarrow}\{0,1\}^{m}$, and computes upk $=\mathbf{p}=\operatorname{bin}(\mathbf{A} \cdot \mathbf{x}) \bmod q \in\{0,1\}^{n k}$.

Without loss of generality, we assume that every honestly generated upk is a non-zero vector. (Otherwise, the user would either pick $\mathbf{x}=\mathbf{0}$ or accidentally find a solution to the $\mathrm{SIS}_{n, m, q, 1}^{\infty}$ problem associated with matrix $\mathbf{A}$ - both happen with negligible probability.)

〈Join, Issue $\rangle$. If the user with key pair (upk, usk) $=(\mathbf{p}, \mathbf{x})$ requests to join the group at epoch $\tau$, he sends $\mathbf{p}$ to GM. If the latter accepts the request, then the two parties proceed as follows.

1. GM issues a member identifier for the user as uid $=\operatorname{bin}(c) \in\{0,1\}^{\ell}$. The user then sets his long-term signing key as gsk $[c]=(\operatorname{bin}(c), \mathbf{p}, \mathbf{x})$.

2. GM performs the following updates:

- Update $\mathcal{T}$ by running algorithm TUpdate $\mathbf{A}_{\mathbf{A}}(\operatorname{bin}(c), \mathbf{p})$. 
- Register the user to table reg as $\operatorname{reg}[c][1]:=\mathbf{p} ; \quad \operatorname{reg}[c][2]:=\tau$.

- Increase the counter $c:=c+1$.

GUpdate(gpk, msk, info $\left.\tau_{\tau_{\text {current }}}, \mathcal{S}, \mathbf{r e g}\right)$. This algorithm is run by GM to update the group information while also advancing the epoch. It works as follows.

1. Let the set $\mathcal{S}$ contain the public keys of registered users to be revoked. If $\mathcal{S}=\emptyset$, then go to Step 2 ,

Otherwise, $\mathcal{S}=\left\{\boldsymbol{r e g}\left[i_{1}\right][1], \ldots, \boldsymbol{r e g}\left[i_{r}\right][1]\right\}$, for some $r \in[1, N]$ and some $i_{1}, \ldots, i_{r} \in[0, N-1]$. Then, for all $t \in[r], \mathrm{GM}$ runs TUpdate $\mathbf{A}_{\mathbf{A}}\left(\operatorname{bin}\left(i_{t}\right), \mathbf{0}^{n k}\right)$ to update the tree $\mathcal{T}$.

2. At this point, by construction, each of the zero leaves in the tree $\mathcal{T}$ corresponds to either a revoked user or a potential user who has not yet registered. In other words, only active users who are allowed to sign in the new epoch $\tau_{\text {new }}$ have their non-zero public keys, denoted by $\left\{\mathbf{p}_{j}\right\}_{j}$, accumulated in the root $\mathbf{u}_{\tau_{\text {new }}}$ of the updated tree.

For each $j$, let $w_{j} \in\{0,1\}^{\ell} \times\left(\{0,1\}^{n k}\right)^{\ell}$ be the witness for the fact that $\mathbf{p}_{j}$ is accumulated in $\mathbf{u}_{\tau_{\text {new }}}$. Then GM publishes the group information of the new epoch as:

$$
\operatorname{info}_{\tau_{\text {new }}}=\left(\mathbf{u}_{\tau_{\text {new }}},\left\{w_{j}\right\}_{j}\right) .
$$

We remark that the info $_{\tau}$ outputted at each epoch by GM is technically not part of the verification key. Indeed, as we will describe below, in order to verify signatures bound to epoch $\tau$, the verifiers only need to download the first component $\mathbf{u}_{\tau}$ of size $\widetilde{\mathcal{O}}(\lambda)$ bits. Meanwhile, each active signer only has to download the respective witness of size $\widetilde{\mathcal{O}}(\lambda) \cdot \ell$.

Sign (gpk, gsk $[j]$, info ${ }_{\tau}, M$ ). To sign message $M$ using the group information info $\tau$ at epoch $\tau$, the user possessing gsk $[j]=(\operatorname{bin}(j), \mathbf{p}, \mathbf{x})$ first checks if info $\tau$ includes a witness containing $\operatorname{bin}(j)$. If this is not the case, return $\perp$. Otherwise, the user downloads $\mathbf{u}_{\tau}$ and the witness of the form $\left(\operatorname{bin}(j),\left(\mathbf{w}_{\ell}, \ldots, \mathbf{w}_{1}\right)\right)$ from info ${ }_{\tau}$, and proceeds as follows.

1. Encrypt vector $\operatorname{bin}(j) \in\{0,1\}^{\ell}$ twice using Regev's encryption scheme.

Namely, for each $i \in\{1,2\}$, sample $\mathbf{r}_{i} \stackrel{\$}{\leftarrow}\{0,1\}^{m_{E}}$ and compute

$$
\begin{aligned}
\mathbf{c}_{i} & =\left(\mathbf{c}_{i, 1}, \mathbf{c}_{i, 2}\right) \\
& =\left(\mathbf{B} \cdot \mathbf{r}_{i} \bmod q, \mathbf{P}_{i} \cdot \mathbf{r}_{i}+\left\lfloor\frac{q}{2}\right\rfloor \cdot \operatorname{bin}(j) \bmod q\right) \in \mathbb{Z}_{q}^{n} \times \mathbb{Z}_{q}^{\ell} .
\end{aligned}
$$

2. Generate a NIZKAoK $\Pi_{\mathrm{gs}}$ to demonstrate the possession of a valid tuple

$$
\zeta=\left(\mathbf{x}, \mathbf{p}, \operatorname{bin}(j), \mathbf{w}_{\ell}, \ldots, \mathbf{w}_{1}, \mathbf{r}_{1}, \mathbf{r}_{2}\right)
$$

such that:

(i) $\operatorname{TVerify}_{\mathbf{A}}\left(\mathbf{u}_{\tau}, \mathbf{p},\left(\operatorname{bin}(j),\left(\mathbf{w}_{\ell}, \ldots, \mathbf{w}_{1}\right)\right)\right)=1$ and $\mathbf{A} \cdot \mathbf{x}=\mathbf{G} \cdot \mathbf{p} \bmod q$;

(ii) $\mathbf{c}_{1}$ and $\mathbf{c}_{2}$ are both correct encryptions of $\operatorname{bin}(j)$ with randomness $\mathbf{r}_{1}$ and $\mathbf{r}_{2}$, respectively;

(iii) $\mathbf{p} \neq \mathbf{0}^{n k}$. 
Note that statements (i) and (ii) were covered by the LLNW protocol 30. Meanwhile, statement (iii) is handled using the technique described at the beginning of this Section. We thus obtain a Stern-like interactive zero-knowledge argument system which is a slight modification of the one from [30]. The detailed description of the protocol is presented in Section 4.3. The protocol is repeated $\kappa=\omega(\log \lambda)$ times to achieve negligible soundness error and made non-interactive via the Fiat-Shamir heuristic as a triple $\Pi_{\mathrm{gs}}=\left(\left\{\mathrm{CMT}_{i}\right\}_{i=1}^{\kappa}, \mathrm{CH},\{\mathrm{RSP}\}_{i=1}^{\kappa}\right)$, where

$$
\mathrm{CH}=\mathcal{H}_{\mathrm{FS}}\left(M,\left(\left\{\mathrm{CMT}_{i}\right\}_{i=1}^{\kappa}, \mathbf{A}, \mathbf{u}_{\tau}, \mathbf{B}, \mathbf{P}_{1}, \mathbf{P}_{2}, \mathbf{c}_{1}, \mathbf{c}_{2}\right) \in\{1,2,3\}^{\kappa} .\right.
$$

3. Output the group signature

$$
\Sigma=\left(\Pi_{\mathrm{gs}}, \mathbf{c}_{1}, \mathbf{c}_{2}\right) .
$$

Verify $\left(\mathrm{gpk}, \mathrm{info}_{\tau}, M, \Sigma\right)$. This algorithm proceeds as follows:

1. Download $\mathbf{u}_{\tau} \in\{0,1\}^{\text {nk }}$ from $\operatorname{info}_{\tau}$.

2. Parse $\Sigma$ as $\Sigma=\left(\left\{\mathrm{CMT}_{i}\right\}_{i=1}^{\kappa},\left(C h_{1}, \ldots, C h_{\kappa}\right),\{\mathrm{RSP}\}_{i=1}^{\kappa}, \mathbf{c}_{1}, \mathbf{c}_{2}\right)$. If $\left(C h_{1}, \ldots, C h_{\kappa}\right) \neq \mathcal{H}_{\mathrm{FS}}\left(M,\left(\left\{\mathrm{CMT}_{i}\right\}_{i=1}^{\kappa}, \mathbf{A}, \mathbf{u}_{\tau}, \mathbf{B}, \mathbf{P}_{1}, \mathbf{P}_{2}, \mathbf{c}_{1}, \mathbf{c}_{2}\right)\right.$, then return 0 .

3. For each $i \in[\kappa]$, run the verification phase of the protocol in Section 4.3 to check the validity of $\mathrm{RSP}_{i}$ with respect to $\mathrm{CMT}_{i}$ and $C h_{i}$. If any of the conditions does not hold, then return 0 .

4. Return 1.

Trace(gpk, tsk, info $\tau$, $\operatorname{reg}, M, \Sigma)$. This algorithm parses tsk as $\left(\mathbf{S}_{1}, \mathbf{E}_{1}\right)$, parses $\Sigma$ as in (3), and performs the following steps.

1. Use $\mathbf{S}_{1}$ to decrypt $\mathbf{c}_{1}=\left(\mathbf{c}_{1,1}, \mathbf{c}_{1,2}\right)$ to obtain a string $\mathbf{b}^{\prime} \in\{0,1\}^{\ell}$ (i.e., by computing $\left.\left\lfloor\frac{\left(\mathbf{c}_{1,2}-\mathbf{S}_{1}^{\top} \cdot \mathbf{c}_{1,1}\right)}{\lfloor q / 2\rfloor}\right\rceil\right)$.

2. If info $\tau$ does not include a witness containing $\mathbf{b}^{\prime}$, then return $\perp$.

3. Let $j^{\prime} \in[0, N-1]$ be the integer having binary representation $\mathbf{b}^{\prime}$. If the record $\operatorname{reg}\left[j^{\prime}\right][1]$ in table reg is $\mathbf{0}^{n k}$, then return $\perp$.

4. Generate a NIZKAoK $\Pi_{\text {trace }}$ to demonstrate the possession of $\mathbf{S}_{1} \in \mathbb{Z}^{n \times \ell}$, $\mathbf{E}_{1} \in \mathbb{Z}^{\ell \times m_{E}}$, and $\mathbf{y} \in \mathbb{Z}^{\ell}$, such that:

$$
\left\{\begin{array}{l}
\left\|\mathbf{S}_{1}\right\|_{\infty} \leq \beta ;\left\|\mathbf{E}_{1}\right\|_{\infty} \leq \beta ;\|\mathbf{y}\|_{\infty} \leq\lceil q / 5\rceil ; \\
\mathbf{S}_{1}^{\top} \cdot \mathbf{B}+\mathbf{E}_{1}=\mathbf{P}_{1} \bmod q ; \\
\mathbf{c}_{1,2}-\mathbf{S}_{1}^{\top} \cdot \mathbf{c}_{1,1}=\mathbf{y}+\lfloor q / 2\rfloor \cdot \mathbf{b}^{\prime} \bmod q .
\end{array}\right.
$$

As the statement involves modular linear equations with bounded-norm secrets, we can obtain a statistical zero-knowledge argument by employing the Stern-like interactive protocol from [28. For completeness, the detailed description of the protocol is presented in Section 4.4. The protocol is repeated $\kappa=\omega(\log \lambda)$ times to achieve negligible soundness error and made non-interactive via the Fiat-Shamir heuristic as a triple $\Pi_{\text {trace }}=\left(\left\{\mathrm{CMT}_{i}\right\}_{i=1}^{\kappa}, \mathrm{CH},\{\mathrm{RSP}\}_{i=1}^{\kappa}\right)$, where

$$
\mathrm{CH}=\mathcal{H}_{\mathrm{FS}}\left(\left(\left\{\mathrm{CMT}_{i}\right\}_{i=1}^{\kappa}, \text { gpk }_{\text {info }}, M, \Sigma, \mathbf{b}^{\prime}\right) \in\{1,2,3\}^{\kappa} .\right.
$$


5. Set uid $=\mathbf{b}^{\prime}$ and output (uid, $\Pi_{\text {trace }}$ ).

Judge(gpk, uid, info $\left.\tau, \Pi_{\text {trace }}, M, \Sigma\right)$. This algorithm consists of verifying the argument $\Pi_{\text {trace }}$ w.r.t. common input (gpk, info $\tau, M, \Sigma$, uid), in a similar manner as in algorithm Verify.

If $\Pi_{\text {trace }}$ does not verify, return 0 . Otherwise, return 1.

\subsection{Analysis of the Scheme}

EFFICIENCY. We first analyze the efficiency of the scheme described in Section 4.1 with respect to security parameter $\lambda$ and parameter $\ell=\log N$.

- The public key gpk contains several matrices, and has bit-size $\widetilde{\mathcal{O}}\left(\lambda^{2}+\lambda \cdot \ell\right)$.

- For each $j \in[0, N-1]$, the signing key gsk $[j]$ has bit-size $\ell+n k+m=\widetilde{\mathcal{O}}(\lambda)+\ell$.

- At each epoch, the signature verifiers downloads $n k=\widetilde{\mathcal{O}}(\lambda)$ bits, while each active signer downloads $\widetilde{\mathcal{O}}(\lambda \cdot \ell)$ bits.

- The size of signature $\Sigma$ is dominated by that of the Stern-like NIZKAoK $\Pi_{\mathrm{gs}}$, which is $\mathcal{O}(|\zeta| \cdot \log q) \cdot \omega(\log \lambda)$, where $|\zeta|$ denotes the bit-size of the witness-tuple $\zeta$ in (2). Overall, $\Sigma$ has bit-size $\widetilde{\mathcal{O}}(\lambda \cdot \ell)$.

- The Stern-like NIZKAoK $\Pi_{\text {trace }}$ has bit-size $\widetilde{\mathcal{O}}\left(\ell^{2}+\lambda \cdot \ell\right)$.

Correctness. We now demonstrate that the scheme is correct with overwhelming probability, based on the perfect completeness of Stern-like protocols, and the correctness of Regev's encryption scheme.

First, note that a signature $\Sigma=\left(\Pi_{\mathrm{gs}}, \mathbf{c}_{1}, \mathbf{c}_{2}\right)$ generated by an active and honest user $j$ is always accepted by algorithm Verify. Indeed, such a user can always compute a tuple $\zeta=\left(\mathbf{x}, \mathbf{p}, \operatorname{bin}(j), \mathbf{w}_{\ell}, \ldots, \mathbf{w}_{1}, \mathbf{r}_{1}, \mathbf{r}_{2}\right)$ satisfying conditions (i) (ii) and (iii) in the Sign algorithm. The completeness of the underlying argument system then guarantees that $\Sigma$ is always accepted by algorithm Verify.

Next, we show that algorithm Trace outputs $\operatorname{bin}(j)$ with overwhelming probability, and produces a proof $\Pi_{\text {trace }}$ accepted by algorithm Judge. Observe that, the decryption algorithm essentially computes

$$
\mathbf{e}=\mathbf{c}_{1,2}-\mathbf{S}_{1}^{T} \mathbf{c}_{1,1}=\mathbf{E}_{1} \cdot \mathbf{r}_{1}+\lfloor q / 2\rfloor \cdot \operatorname{bin}(j) \bmod q,
$$

and sets the $j$-th bit of $\mathbf{b}^{\prime}$ to be 0 if $j$-th entry of $\mathbf{e}$ is closer to 0 than to $\lfloor q / 2\rfloor$ and 1 otherwise. Note that our parameters are set so that $\left\|\mathbf{E}_{1} \cdot \mathbf{r}_{1}\right\|_{\infty}<$ $q / 5$, for $\mathbf{E}_{1} \hookleftarrow \chi^{\ell \times m_{E}}$ and $\mathbf{r}_{1} \stackrel{\$}{\leftarrow}\{0,1\}^{m_{E}}$. This ensures that $\mathbf{b}^{\prime}=\operatorname{bin}(j)$ with overwhelming probability.

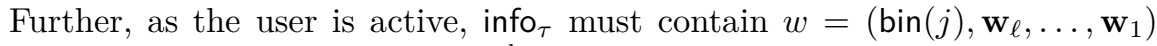
and $\operatorname{reg}[j][1]$ in table $\mathbf{r e g}$ is not $\mathbf{0}^{n k}$. Therefore, algorithm Trace will move to the 4-th step, where it can always obtain the tuple $\left(\mathbf{S}_{1}, \mathbf{E}_{1}, \mathbf{y}\right)$ satisfying the conditions in (4). By the completeness of the argument system, $\Pi_{\text {trace }}$ will be accepted by the algorithm Judge.

SECURITY. In Theorem 1 we prove that our scheme satisfies the security requirements of the Bootle et al.'s model [7]. 
Theorem 1. Assume that the Stern-like argument systems used in Section 4.1 are simulation-sound. Then, in the random oracle model, the given fully dynamic group signature satisfies the anonymity, traceability, non-frameability and tracing soundness requirements under the $\mathrm{LWE}_{n, q, \chi}$ and $\mathrm{SIS}_{n, m, q, 1}^{\infty}$ assumptions.

In the random oracle model, the proof of Theorem 1 relies on the following facts:

1. The Stern-like zero-knowledge argument systems being used are simulationsound;

2. The underlying encryption scheme, which is obtained from Regev cryptosystem [45] via the Naor-Yung transformation 41, is IND-CCA2 secure;

3. The Merkle tree accumulator we employ is secure in the sense of Definition 4 :

4. For a properly generated key-pair $(\mathbf{x}, \mathbf{p})$, it is infeasible to find $\mathbf{x}^{\prime} \in\{0,1\}^{m}$ such that $\mathbf{x}^{\prime} \neq \mathbf{x}$ and $\operatorname{bin}\left(\mathbf{A} \cdot \mathbf{x}^{\prime} \bmod q\right)=\mathbf{p}$.

The proof of Theorem 1 is established by Lemmas 2 2 given below.

Lemma 2. Assume that the $\mathrm{LWE}_{n, q, \chi}$ problem is hard. Then the given FDGS scheme provides anonymity in the random oracle model.

Proof. Let $\mathcal{A}, \mathcal{B}$ be PPT algorithms, act as the adversary and challenger in the games respectively. We construct a sequence of indistinguishable games, in which the first game is the experiment $\operatorname{Exp}_{\mathrm{FDGS}, \mathcal{A}}^{\text {anon-0 }}(\lambda)$ and the last one is the experiment $\operatorname{Exp}_{\mathrm{FDGS}, \mathcal{A}}^{\text {anon }-1}(\lambda)$. We will prove the lemma by demonstrating that any two consecutive games are indistinguishable. For each $i$, we denote by $W_{i}$ the output of the adversary in game $i$.

Specifically, we consider the following games.

Game 0: This is exactly the experiment $\operatorname{Exp}_{\mathrm{FDGS}, \mathcal{A}}^{\mathrm{anon}-0}(\lambda)$.

Game 1: This game is the same as Game 0 with only one modification: add $\left(\mathbf{S}_{2}, \mathbf{E}_{2}\right)$ to tsk. This will make no difference to the view of the adversary. So $\operatorname{Pr}\left[W_{1}=1\right]=\operatorname{Pr}\left[\operatorname{Exp}_{\mathrm{FDGS}, \mathcal{A}}^{\mathrm{anon}-0}(\lambda)=1\right]$.

Game 2: This game is identical to Game 1 except that it generates a simulated proof for the oracle Trace by programming the random oracle $\mathcal{H}_{\mathrm{FS}}$ even though the challenger $\mathcal{B}$ has correct witnesses to generate a real proof. The view of the adversary, however, is statistically indistinguishable between Game 1 and Game 2 by statistical zero-knowledge property of our argument system generating $\Pi_{\text {trace }}$. Therefore we have $\operatorname{Pr}\left[W_{1}=1\right] \approx \operatorname{Pr}\left[W_{2}=1\right]$.

Game 3: This game uses $\mathbf{S}_{2}$ instead of $\mathbf{S}_{1}$ to simulate the oracle Trace. The view of $\mathcal{A}$ is the same as in Game 2 until event $F_{1}$, where $\mathcal{A}$ queries the trace oracle a valid signature $\left(M, \Pi_{\mathrm{gs}}, \mathbf{c}_{1}, \mathbf{c}_{2}\right.$, info $\left._{\tau}\right)$ with $\mathbf{c}_{1}, \mathbf{c}_{2}$ encrypting distinct bit strings, happens. Since $F_{1}$ breaks the soundness of our argument generating $\Pi_{\mathrm{gs}}$, we have $\left|\operatorname{Pr}\left[W_{2}=1\right]-\operatorname{Pr}\left[W_{3}=1\right]\right| \leq \operatorname{Pr}\left[F_{1}\right] \leq \operatorname{Adv}_{\Pi_{\mathrm{gs}}}^{\text {sound }}(\lambda)=\operatorname{negl}(\lambda)$.

Game 4: This game generates a simulated proof for the oracle Chal. The view is indistinguishable to $\mathcal{A}$ by statistical zero-knowledge property of our argument system generating $\Pi_{\mathrm{gs}}$. Therefore we have $\operatorname{Pr}\left[W_{3}=1\right] \approx \operatorname{Pr}\left[W_{4}=1\right]$. 
Game 5: This is the same as Game 4 except that $\mathbf{c}_{1}$ is now encryption of $\operatorname{bin}\left(i_{1}\right)$ while $\mathbf{c}_{2}$ is still encryption of $\operatorname{bin}\left(i_{0}\right)$ for the Chal query. By the semantic security of our encryption scheme for public key $\left(\mathbf{B}, \mathbf{P}_{1}\right)$ (which relies on LWE assumption), this change is negligible to the adversary. Recall that we use $\mathbf{S}_{2}$ for the Trace queries. So the change of $\mathbf{c}_{1}$ makes no difference to the view of the adversary. Therefore we have $\left|\operatorname{Pr}\left[W_{4}=1\right]-\operatorname{Pr}\left[W_{5}=1\right]\right|=\operatorname{negl}(\lambda)$.

Game 6: This game follows Game 5 with one change: it switches back to use $\mathbf{S}_{1}$ for the Trace queries and discards $\left(\mathbf{S}_{2}, \mathbf{E}_{2}\right)$. This modification is indistinguishable to the adversary until event $F_{2}$, where $\mathcal{A}$ queries the trace oracle a valid signature $\left(M, \Pi_{\mathrm{gs}}, \mathbf{c}_{1}, \mathbf{c}_{2}\right.$, info $\left._{\tau}\right)$ with $\mathbf{c}_{1}, \mathbf{c}_{2}$ encrypting different bit strings, happens. However event $F_{2}$ breaks the simulation soundness of the argument system generating $\Pi_{\mathrm{gs}}$. Therefore we have $\left|\operatorname{Pr}\left[W_{5}=1\right]-\operatorname{Pr}\left[W_{6}=1\right]\right| \leq$ $\operatorname{Adv}_{I_{\mathrm{gs}}}^{\mathrm{ss}}(\lambda)=\operatorname{negl}(\lambda)$.

Game 7: In this game, it changes $\mathbf{c}_{2}$ to be encryption of $\operatorname{bin}\left(i_{1}\right)$ for the Chal query while the remaining parts are the same as in Game 6. By the semantic security of our encryption scheme for public key $\left(\mathbf{B}, \mathbf{P}_{2}\right)$, this change is negligible to the adversary. Note that we now use $\mathbf{S}_{1}$ for the Trace queries. So changing $\mathbf{c}_{2}$ makes no difference to the view of the adversary. Therefore we have $\left|\operatorname{Pr}\left[W_{6}=1\right]-\operatorname{Pr}\left[W_{7}=1\right]\right|=\operatorname{negl}(\lambda)$.

Game 8: We now generate real proof for the Chal query instead of simulated proof. By the statistical zero-knowledge property of our argument system generating $\Pi_{\mathrm{gs}}$, the view of the adversary in Game 7 and Game 8 are statistically indistinguishable, i.e., we have $\operatorname{Pr}\left[W_{7}=1\right] \approx \operatorname{Pr}\left[W_{8}=1\right]$.

Game 9: This game produces real proof, for the Trace queries. By the statistical zero-knowledge property of our argument system generating $\Pi_{\text {trace }}$, we have Game 8 and Game 9 are statistically indistinguishable to the adversary, i.e., we have $\operatorname{Pr}\left[W_{8}=1\right] \approx \operatorname{Pr}\left[W_{9}=1\right]$. This is actually the experiment $\operatorname{Exp}_{\mathrm{FDGS}, \mathcal{A}}^{\text {anon }-1}(\lambda)$. Hence, we have $\operatorname{Pr}\left[W_{9}=1\right]=\operatorname{Pr}\left[\operatorname{Exp}_{\mathrm{FDGS}, \mathcal{A}}^{\text {anon }-1}(\lambda)=1\right]$.

As a result, we have that

$$
\left|\operatorname{Pr}\left[\operatorname{Exp}_{\mathrm{FDGS}, \mathcal{A}}^{\mathrm{anon}-1}(\lambda)=1\right]-\operatorname{Pr}\left[\operatorname{Exp}_{\mathrm{FDGS}, \mathcal{A}}^{\mathrm{anon}-0}(\lambda)=1\right]\right|=\operatorname{negl}(\lambda),
$$

and hence our scheme is anonymous.

Lemma 3. Assume that the $\mathrm{SIS}_{n, m, q, 1}^{\infty}$ problem is hard. Then the given FDGS scheme provides non-frameability in the random oracle model.

Proof. We prove non-frameability by contradiction. Suppose that $\mathcal{A}$ succeeds with non-negligible advantage $\epsilon$. Then we can build a PPT algorithm $\mathcal{B}$ that either breaks the security of our accumulator or solves $\mathrm{SIS}_{n, q, m, 1}^{\infty}$ problem, which are featured by $\mathbf{A}$, with also non-negligible probability.

Given a matrix $\mathbf{A}$ from the environment that $\mathcal{B}$ is in, it first generates all parameters pp as we do in GSetup, then invokes $\mathcal{A}$ with pp, and then proceeds as described in the experiment. Here $\mathcal{B}$ can consistently answer all the oracle 
queries made by $\mathcal{A}$. When $\mathcal{A}$ outputs $\left(M^{*}, \Sigma^{*}, \operatorname{bin}\left(j^{*}\right), \Pi_{\text {trace }}^{*}\right.$, info $\left.\tau\right)$ and wins, $\mathcal{B}$ does following.

Parse $\Sigma^{*}$ as $\left(\Pi_{\mathrm{gs}}^{*}, \mathbf{c}_{1}^{*}, \mathbf{c}_{2}^{*}\right)$, where $\Pi_{\mathrm{gs}}^{*}=\left(\left\{\mathrm{CMT}_{i}^{*}\right\}_{i=1}^{\kappa}, \mathrm{CH}^{*},\left\{\mathrm{RSP}_{i}^{*}\right\}_{i=1}^{\kappa}\right)$ and $\mathrm{RSP}_{i}^{*}$ is a valid response w.r.t $\mathrm{CMT}_{i}^{*}$ and $\mathrm{CH}_{i}^{*}$ for $i \in[\kappa]$ since $\mathcal{A}$ wins. We claim that $\mathcal{A}$ queried the tuple $\left(M^{*},\left\{\mathrm{CMT}_{i}^{*}\right\}_{i=1}^{k}, \mathbf{A}, \mathbf{u}_{\tau}, \mathbf{B}, \mathbf{P}_{1}, \mathbf{P}_{2}, \mathbf{c}_{1}^{*}, \mathbf{c}_{2}^{*}\right)$, denoted as $\xi^{*}$, to the hash oracle $\mathcal{H}_{\mathrm{FS}}$ with overwhelming probability. Otherwise guessing correctly this value occurs only with probability $3^{-\kappa}$, which is negligible. Therefore, with probability $\epsilon^{\prime}=\epsilon-3^{-\kappa}$, the tuple $\xi^{*}$ has been an input of one hash query, denoted as $t^{*} \in\left\{1,2 \cdots, Q_{H}\right\}$, where $Q_{H}$ is the total number of hash queries made by $\mathcal{A}$. To employ the Forking lemma of Brickell et al. 11], $\mathcal{B}$ runs polynomial-number executions of $\mathcal{A}$ exactly the same as in the original run until the $t^{*}$ hash query, that is, from $t^{*}$ hash query on, $\mathcal{B}$ will answer $\mathcal{A}$ with fresh and independent values for hash queries on each new execution. Note that the input of $t^{*}$ hash query must be $\xi^{*}$ as in the original run. By the Forking Lemma [11], with probability $\geq \frac{1}{2}, \mathcal{B}$ obtains 3 -fork involving the same tuple $\xi^{*}$ with pairwise distinct hash value $\mathrm{CH}_{t^{*}}^{(1)}, \mathrm{CH}_{t^{*}}^{(2)}, \mathrm{CH}_{t^{*}}^{(3)} \in\{1,2,3\}^{\kappa}$. We have $\left(\mathrm{CH}_{t^{*}, j}^{(1)}, \mathrm{CH}_{t^{*}, j}^{(2)}, \mathrm{CH}_{t^{*}, j}^{(3)}\right)=(1,2,3)$ with probability $1-\left(\frac{7}{9}\right)^{\kappa}$ for some $j \in\{1,2, \ldots, \kappa\}$. Then by the argument of knowledge of the system generating $\Pi_{\mathrm{gs}}$, from the valid responses $\left(\mathrm{RSP}_{t^{*}, j}^{(1)}, \mathrm{RSP}_{t^{*}, j}^{(2)}, \mathrm{RSP}_{t^{*}, j}^{(3)}\right)$, we can extract the witnesses $\zeta^{\prime}=\left(\mathbf{x}^{\prime}, \mathbf{p}^{\prime}, w_{\tau}^{\prime}, \mathbf{r}_{1}^{\prime}, \mathbf{r}_{2}^{\prime}\right)$, where

$$
w_{\tau}^{\prime}=\left(\operatorname{bin}\left(j^{\prime}\right), \mathbf{w}_{\ell, \tau}^{\prime}, \ldots, \mathbf{w}^{\prime}{ }_{1, \tau}\right) \in\{0,1\}^{\ell} \times\left(\{0,1\}^{n k}\right)^{\ell},
$$

such that we have:

(i) $\operatorname{TVerify}_{\mathbf{A}}\left(\mathbf{u}_{\tau}, \mathbf{p}^{\prime}, w_{\tau}^{\prime}\right)=1, \mathbf{p}^{\prime} \neq 0$;

(ii) $\mathbf{A} \cdot \mathbf{x}^{\prime}=\mathbf{G} \cdot \mathbf{p}^{\prime} \bmod q$;

(iii) $\mathbf{c}_{1}^{*}, \mathbf{c}_{2}^{*}$ are encryptions of $\operatorname{bin}\left(j^{\prime}\right)$ with randomness $\mathbf{r}_{1}^{\prime}$ and $\mathbf{r}_{2}^{\prime}$.

By the correctness of our encryption scheme, $\mathbf{c}_{1}^{*}$ is decrypted to $\operatorname{bin}\left(j^{\prime}\right)$. The fact $\mathcal{A}$ wins implies algorithm Judge outputs 1 . By the soundness of our system generating $\Pi_{\text {trace }}$, bin $\left(j^{*}\right)$ is decrypted from $\mathbf{c}_{1}^{*}$. Hence we have $\operatorname{bin}\left(j^{\prime}\right)=\operatorname{bin}\left(j^{*}\right)$ with overwhelming probability.

Now we consider the following two cases:

Case 1: $\operatorname{bin}\left(j^{\prime}\right)$ is a inactive user at epoch $\tau$, i.e., the value at leaf $\operatorname{bin}\left(j^{\prime}\right)$ is $\mathbf{0}^{n k}$. This violates the accumulator security since we have $\operatorname{TVerify}_{\mathbf{A}}\left(\mathbf{u}_{\tau}, \mathbf{p}^{\prime}, w_{\tau}^{\prime}\right)=1$ with $\mathbf{p}^{\prime} \neq \mathbf{0}$ at leaf $\operatorname{bin}\left(j^{\prime}\right)$.

Case 2: $\operatorname{bin}\left(j^{*}\right)$ is an active user at epoch $\tau$. The fact $\operatorname{bin}\left(j^{*}\right) \in$ HUL $\backslash$ BUL indicates $\mathcal{A}$ does not know $\operatorname{gsk}\left[j^{*}\right]=\left(\operatorname{bin}\left(j^{*}\right), \mathbf{p}^{\prime}, \mathbf{x}_{j^{*}}\right)$, where $\mathbf{x}_{j^{*}}$ was initially chosen by $\mathcal{B}$ and satisfies $\mathbf{A} \cdot \mathbf{x}_{j^{*}}=\mathbf{G} \cdot \mathbf{p}^{\prime} \bmod q$. Following the same argument of [30], we claim that $\mathbf{x}_{j^{*}} \neq \mathbf{x}^{\prime}$ with probability at least $1 / 2$. Then we find a nonzero vector $\mathbf{z}=\mathbf{x}_{j^{*}}-\mathbf{x}^{\prime}$ satisfying $\mathbf{A} \mathbf{z}=0 \bmod q$. Recall $\mathbf{x}_{j^{*}}, \mathbf{x}^{\prime} \in\{0,1\}^{m}$. Thus $\mathcal{B}$ solves a $\operatorname{SIS}_{n, q, m, 1}^{\infty}$ problem with non-negligible probability if $\mathcal{A}$ breaks the non-frameability of our construction with non-negligible probability.

Lemma 4. Assume that the $\mathrm{SIS}_{n, m, q, 1}^{\infty}$ problem is hard. Then the given FDGS scheme satisfies traceability in the random oracle model. 
Proof. Recall that adversary $\mathcal{A}$ wins traceability experiment if it generates a valid signature that either: (i) traces to an inactive user; or (ii) traces to an active user but we cannot generate a proof accepted by the algorithm Judge. We will prove that both cases occur with negligible probability.

Let ( info $_{\tau}, M, \Sigma$ ) be the output of $\mathcal{A}$ in the experiment $\operatorname{Exp}_{\mathrm{FDGS}, \mathcal{A}}^{\text {trace }}(\lambda)$ and we compute (bin $\left.(j), \Pi_{\text {trace }}\right)$ by running the algorithm Trace. Parse $\Sigma$ as $\left(\Pi_{\mathrm{gs}}, \mathbf{c}_{1}, \mathbf{c}_{2}\right)$, where $\Pi_{\mathrm{gs}}=\left(\left\{\mathrm{CMT}_{i}\right\}_{i=1}^{\kappa}, \mathrm{CH},\left\{\mathrm{RSP}_{i}\right\}_{i=1}^{\kappa}\right)$, and $\mathrm{RSP}_{i}$ is a valid response w.r.t. $\mathrm{CMT}_{i}$ and $\mathrm{CH}_{i}$ for $i \in[\kappa]$ since ( info $_{\tau}, M, \Sigma$ ) is a valid signature outputted by $\mathcal{A}$. Now we are in the same situation as in Lemma 3 By the same technique, we can extract witnesses $\zeta=\left(\mathbf{x}, \mathbf{p}, w_{\tau}, \mathbf{r}_{1}, \mathbf{r}_{2}\right)$, where $w_{\tau}=\left(\operatorname{bin}\left(j^{\prime}\right), \mathbf{w}_{\ell, \tau}, \ldots, \mathbf{w}_{1, \tau}\right) \in$ $\{0,1\}^{\ell} \times\left(\{0,1\}^{n k}\right)^{\ell}$ satisfying TVerify $\mathbf{A}\left(\mathbf{u}_{\tau}, \mathbf{p}, w_{\tau}\right)=1, \mathbf{p} \neq \mathbf{0}^{n k}$, and $\mathbf{c}_{1}, \mathbf{c}_{2}$ are encryptions of $\operatorname{bin}\left(j^{\prime}\right)$ using randomness $\mathbf{r}_{1}$ and $\mathbf{r}_{2}$. Correctness of our encryption scheme implies $\mathbf{c}_{1}$ is decrypted to $\operatorname{bin}\left(j^{\prime}\right)$. Correct decryption of the Trace algorithm, which is run by the challenger, indicates that $\operatorname{bin}(j)$ is decrypted from $\mathbf{c}_{1}$. Hence we have $\operatorname{bin}\left(j^{\prime}\right)=\operatorname{bin}(j)$ with overwhelming probability.

Now, we note that case (i) considered above only happens with negligible probability. In fact, if $\operatorname{bin}(j)$ is not active at epoch $\tau$, and $\operatorname{TVerify}_{\mathbf{A}}\left(\mathbf{u}_{\tau}, \mathbf{p}, w_{\tau}\right)=1$ with $\mathbf{p} \neq \mathbf{0}$ at leaf $\operatorname{bin}(j)$, then the security of our accumulator is violated. Furthermore, case (ii) also occurs with negligible probability since the challenger possesses valid witnesses to generate the proof $\Pi_{\text {trace }}$, which will be accepted by the Judge algorithm, thanks to the completeness of the underlying argument system. It then follows that our scheme satisfies the traceability requirement.

Lemma 5. The given FDGS scheme satisfies tracing soundness in the random oracle model.

Proof. Let $\left(M, \Sigma, \operatorname{bin}\left(j_{0}\right), \Pi_{\text {trace }, 0}, \operatorname{bin}\left(j_{1}\right), \Pi_{\text {trace }, 1}, \operatorname{info}_{\tau}\right)$ be the output of the adversary in the experiment $\operatorname{Exp}_{\mathrm{FDGS}, \mathcal{A}}^{\text {trace-sound }}(\lambda)$. Recall that adversary wins if the following conditions hold:

(i) $\operatorname{bin}\left(j_{0}\right) \neq \operatorname{bin}\left(j_{1}\right)$;

(ii) $\operatorname{Verify}\left(\mathrm{gpk}\right.$ info $\left._{\tau}, M, \Sigma\right)=1$;

(iii) Judge $\left(\mathrm{gpk}, \operatorname{bin}\left(j_{b}\right)\right.$, info $\left._{\tau}, \Pi_{\text {trace }, b}, M, \Sigma\right)=1$ for $b \in\{0,1\}$;

(iv) $\operatorname{bin}\left(j_{b}\right) \neq \perp$ for $b \in\{0,1\}$.

Let us parse $\Pi_{\text {trace }, b}$ as $\left(\left\{\mathrm{CMT}_{i, b}\right\}_{i=1}^{\kappa}, \mathrm{CH}_{b},\left\{\mathrm{RSP}_{i, b}\right\}_{i=1}^{\kappa}\right)$. The fact that the Judge algorithm outputs 1 implies that $\mathrm{RSP}_{i, b}$ is a valid response w.r.t. $\mathrm{CMT}_{i, b}$ and $\mathrm{CH}_{i, b}$ for $i \in[\kappa]$ and $b \in\{0,1\}$. By using the extraction technique for argument system generating $\Pi_{\text {trace }}$ as in Lemma 3, we can extract the witnesses $\mathbf{S}_{1, b}, \mathbf{E}_{1, b}, \mathbf{y}_{b}$ for $b \in\{0,1\}$ satisfying:

$$
\left\{\begin{array}{l}
\left\|\mathbf{S}_{1, b}\right\|_{\infty} \leq \beta ;\left\|\mathbf{E}_{1, b}\right\|_{\infty} \leq \beta ;\left\|\mathbf{y}_{b}\right\|_{\infty} \leq\lceil q / 5\rceil ; \\
\mathbf{S}_{1, b}^{\top} \cdot \mathbf{B}+\mathbf{E}_{1, b}=\mathbf{P}_{1} \bmod q ; \\
\mathbf{c}_{1,2}-\mathbf{S}_{1, b}^{\top} \cdot \mathbf{c}_{1,1}=\mathbf{y}_{b}+\lfloor q / 2\rfloor \cdot \operatorname{bin}\left(j_{b}\right) \bmod q .
\end{array}\right.
$$


Note that, we have $\left(\mathbf{S}_{1,0}^{\top}-\mathbf{S}_{1,1}^{\top}\right) \cdot \mathbf{c}_{1,1}=\mathbf{y}_{1}-\mathbf{y}_{0}+\lfloor q / 2\rfloor \cdot\left(\operatorname{bin}\left(j_{1}\right)-\operatorname{bin}\left(j_{0}\right)\right) \bmod q$. Suppose that $\operatorname{bin}\left(j_{0}\right) \neq \operatorname{bin}\left(j_{1}\right)$, then $\left\|\lfloor q / 2\rfloor \cdot\left(\operatorname{bin}\left(j_{1}\right)-\operatorname{bin}\left(j_{0}\right)\right)\right\|_{\infty}=\lfloor q / 2\rfloor$. On the other hand, we have $\left\|\mathbf{y}_{1}-\mathbf{y}_{0}\right\|_{\infty} \leq 2 \cdot\lceil q / 5\rceil$. It then follows that

$$
\left\|\mathbf{y}_{1}-\mathbf{y}_{0}+\lfloor q / 2\rfloor \cdot\left(\operatorname{bin}\left(j_{1}\right)-\operatorname{bin}\left(j_{0}\right)\right)\right\|_{\infty}>0,
$$

which implies that $\mathbf{S}_{1,0}^{\top} \neq \mathbf{S}_{1,1}^{\top}$. In other words, we can obtain two solutions for the equation $\mathbf{S}^{\top} \cdot \mathbf{B}+\mathbf{E}=\mathbf{P}_{1} \bmod q$, which contradicts the fact that there exists at most one solution for LWE samples $\left(\mathbf{B}, \mathbf{P}_{1}\right)$.

By contradiction, we have $\operatorname{bin}\left(j_{0}\right)=\operatorname{bin}\left(j_{1}\right)$ with overwhelming probability. This implies that case (i) does not hold with overwhelming probability once cases (ii),(iii) and (iv) hold. As a result, the advantage of the adversary attacking the tracing soundness of our scheme is negligible (in $\lambda$ ). In other words, our construction satisfies tracing soundness.

\subsection{Details of the Main Zero-Knowledge Argument System}

Our protocol is a modification of the one from [30, in which we additionally prove that $\mathbf{p} \neq \mathbf{0}^{n k}$ using the technique discussed at the beginning of Section 4 . We will employ a new strategy for Stern-like protocols, suggested in [28, which consists of unifying all the equations to be proved into just one equation of the form $\mathbf{M} \cdot \mathbf{z}=\mathbf{u} \bmod q$, for some public matrix $\mathbf{M}$ and vector $\mathbf{u}$ over $\mathbb{Z}_{q}$. This strategy will help us to deliver a simpler presentation than in [30].

Let us first examine the equations associated with the execution of algorithm $\operatorname{TVerify}_{\mathbf{A}}\left(\mathbf{u}_{\tau}, \mathbf{p},\left(\left(j_{1}, \ldots, j_{\ell}\right),\left(\mathbf{w}_{\ell}, \ldots, \mathbf{w}_{1}\right)\right)\right)$. This algorithm computes the tree path $\mathbf{v}_{\ell}, \mathbf{v}_{\ell-1}, \ldots, \mathbf{v}_{1}, \mathbf{v}_{0} \in\{0,1\}^{n k}$ as follows: $\mathbf{v}_{\ell}=\mathbf{p}$ and

$$
\forall i \in\{\ell-1, \ldots, 1,0\}: \mathbf{v}_{i}=\left\{\begin{array}{l}
h_{\mathbf{A}}\left(\mathbf{v}_{i+1}, \mathbf{w}_{i+1}\right), \text { if } j_{i+1}=0 \\
h_{\mathbf{A}}\left(\mathbf{w}_{i+1}, \mathbf{v}_{i+1}\right), \text { if } j_{i+1}=1 .
\end{array}\right.
$$

The algorithm outputs 1 if $\mathbf{v}_{0}=\mathbf{u}_{\tau}$. Observe that relation (7) can be equivalently rewritten as: $\forall i \in\{\ell-1, \ldots, 1,0\}$,

$$
\begin{aligned}
& \bar{j}_{i+1} \cdot h_{\mathbf{A}}\left(\mathbf{v}_{i+1}, \mathbf{w}_{i+1}\right)+j_{i+1} \cdot h_{\mathbf{A}}\left(\mathbf{w}_{i+1}, \mathbf{v}_{i+1}\right)=\mathbf{v}_{i} \\
\Leftrightarrow & \bar{j}_{i+1} \cdot\left(\mathbf{A}_{0} \cdot \mathbf{v}_{i+1}+\mathbf{A}_{1} \cdot \mathbf{w}_{i+1}\right)+j_{i+1} \cdot\left(\mathbf{A}_{0} \cdot \mathbf{w}_{i+1}+\mathbf{A}_{1} \cdot \mathbf{v}_{i+1}\right)=\mathbf{G} \cdot \mathbf{v}_{i} \bmod q \\
\Leftrightarrow & \mathbf{A} \cdot\left(\begin{array}{l}
\bar{j}_{i+1} \cdot \mathbf{v}_{i+1} \\
j_{i+1} \cdot \mathbf{v}_{i+1}
\end{array}\right)+\mathbf{A} \cdot\left(\begin{array}{c}
j_{i+1} \cdot \mathbf{w}_{i+1} \\
\bar{j}_{i+1} \cdot \mathbf{w}_{i+1}
\end{array}\right)=\mathbf{G} \cdot \mathbf{v}_{i} \bmod q \\
\Leftrightarrow & \mathbf{A} \cdot \operatorname{ext}\left(j_{i+1}, \mathbf{v}_{i+1}\right)+\mathbf{A} \cdot \operatorname{ext}\left(\bar{j}_{i+1}, \mathbf{w}_{i+1}\right)=\mathbf{G} \cdot \mathbf{v}_{i} \bmod q .
\end{aligned}
$$

In the above, we use the notation $\operatorname{ext}(b, \mathbf{v})$, for bit $b$ and vector $\mathbf{v}$, to denote the vector $\left(\begin{array}{l}\bar{b} \cdot \mathbf{v} \\ b \cdot \mathbf{v}\end{array}\right)$. Later on, we will also use the notation $\operatorname{ext}_{2}(b)$, for bit $b$, to denote the 2-dimensional vector $(\bar{b}, b)^{\top}$.

Given the above discussion, our protocol can be summarized as follows. 
Public input: A, G, $\mathbf{u}_{\tau}, \mathbf{B}, \mathbf{P}_{1}, \mathbf{P}_{2}, \mathbf{c}_{1}=\left(\mathbf{c}_{1,1}, \mathbf{c}_{1,2}\right), \mathbf{c}_{2}=\left(\mathbf{c}_{2,1}, \mathbf{c}_{2,2}\right)$.

Prover's goal: Proving knowledge of secret $\mathbf{x} \in\{0,1\}^{m}, \mathbf{0} \neq \mathbf{p} \in\{0,1\}^{n k}$, $j_{1}, \ldots, j_{\ell} \in\{0,1\}, \mathbf{v}_{1}, \ldots, \mathbf{v}_{\ell-1}, \mathbf{w}_{1}, \ldots, \mathbf{w}_{\ell} \in\{0,1\}^{n k}, \mathbf{r}_{1}, \mathbf{r}_{2} \in\{0,1\}^{m_{E}}$, such that the following equations hold:

$$
\left\{\begin{array}{l}
\mathbf{A} \cdot \operatorname{ext}\left(j_{1}, \mathbf{v}_{1}\right)+\mathbf{A} \cdot \operatorname{ext}\left(\bar{j}_{1}, \mathbf{w}_{1}\right)=\mathbf{G} \cdot \mathbf{u}_{\tau} \bmod q ; \\
\mathbf{A} \cdot \operatorname{ext}\left(j_{2}, \mathbf{v}_{2}\right)+\mathbf{A} \cdot \operatorname{ext}\left(\bar{j}_{2}, \mathbf{w}_{2}\right)-\mathbf{G} \cdot \mathbf{v}_{1}=\mathbf{0} \bmod q ; \\
\quad \ldots \ldots \ldots \\
\mathbf{A} \cdot \operatorname{ext}\left(j_{\ell}, \mathbf{p}\right)+\mathbf{A} \cdot \operatorname{ext}\left(\bar{j}_{\ell}, \mathbf{w}_{\ell}\right)-\mathbf{G} \cdot \mathbf{v}_{\ell-1}=\mathbf{0} \bmod q ; \\
\mathbf{A} \cdot \mathbf{x}-\mathbf{G} \cdot \mathbf{p}=\mathbf{0} \bmod q ; \\
\mathbf{B} \cdot \mathbf{r}_{b}=\mathbf{c}_{b, 1} \bmod q, \text { for } b \in\{1,2\} ; \\
\mathbf{P}_{b} \cdot \mathbf{r}_{b}+\left\lfloor\frac{q}{2}\right\rceil \cdot\left(j_{1}, \ldots, j_{\ell}\right)^{\top}=\mathbf{c}_{b, 2} \bmod q, \text { for } b \in\{1,2\} .
\end{array}\right.
$$

Now, we employ extending-then-permuting techniques for Stern-like protocols 493513028 to handle the secret objects, as follows. For any positive integer $i$, we let $\mathcal{S}_{i}$ denote the set of all permutations of $i$ elements.

- To prove that $\mathbf{p} \in\{0,1\}^{n k}$ and $\mathbf{p} \neq 0^{n k}$, we append $n k-1$ "dummy" entries to $\mathbf{p}$ to get $\mathbf{p}^{*} \in \mathrm{B}_{n k}^{2 n k-1}$. Note that, for any $\pi_{p} \in \mathcal{S}_{2 n k-1}$, we have:

$$
\mathbf{p}^{*} \in \mathrm{B}_{n k}^{2 n k-1} \Leftrightarrow \pi_{p}\left(\mathbf{p}^{*}\right) \in \mathrm{B}_{n k}^{2 n k-1} .
$$

- To prove that $\mathbf{x} \in\{0,1\}^{m}$, we append $m$ "dummy" entries to $\mathbf{x}$ to get $\mathbf{x}^{*} \in \mathrm{B}_{m}^{2 m}$. Note that, for any $\pi_{x} \in \mathcal{S}_{2 m}$, we have: $\mathbf{x}^{*} \in \mathrm{B}_{m}^{2 m} \Leftrightarrow \pi_{x}\left(\mathbf{x}^{*}\right) \in \mathrm{B}_{m}^{2 m}$.

- Similarly, we extend $\mathbf{v}_{1}, \ldots, \mathbf{v}_{\ell-1}, \mathbf{w}_{1}, \ldots, \mathbf{w}_{\ell}$ to $\mathbf{v}_{1}^{*}, \ldots, \mathbf{v}_{\ell-1}^{*}, \mathbf{w}_{1}^{*}, \ldots, \mathbf{w}_{\ell}^{*} \in$ $\mathrm{B}_{n k}^{2 n k}$, respectively. We also extend $\mathbf{r}_{1}, \mathbf{r}_{2}$ to $\mathbf{r}_{1}^{*}, \mathbf{r}_{2}^{*} \in \mathrm{B}_{m_{E}}^{2 m_{E}}$, respectively.

- For each $i=1, \ldots, \ell$, to prove that the bit $j_{i} \in\{0,1\}$ is involved in both encryption layer and Merkle tree layer, we extend it to $\mathbf{j}_{i}=\operatorname{ext}_{2}\left(j_{i}\right)$. Then we use the following permuting technique.

For bit $b \in\{0,1\}$, we let $T_{b}$ be the permutation that transforms vector $\mathbf{t}=\left(t_{0}, t_{1}\right)^{\top} \in \mathbb{Z}^{2}$ into vector $T_{b}(\mathbf{t})=\left(t_{b}, t_{\bar{b}}\right)$. Note that, for any $b_{i} \in$ $\{0,1\}$, we have: $\mathbf{j}_{i}=\operatorname{ext}_{2}\left(j_{i}\right) \Leftrightarrow T_{b_{i}}\left(\mathbf{j}_{i}\right)=\operatorname{ext}_{2}\left(j_{i} \oplus b_{i}\right)$, where $\oplus$ denotes the addition modulo 2 . Then, to prove knowledge of $j_{i} \in\{0,1\}$, we instead prove knowledge of $\mathbf{j}_{i}=\operatorname{ext}_{2}\left(j_{i}\right)$, by sampling $b_{i} \stackrel{\$}{\leftarrow}\{0,1\}$ and showing the verifier that the right-hand side of the equivalence holds. Here, the crucial point is that $b_{i}$ acts as a "one-time pad" that perfectly hides $j_{i}$. Furthermore, to prove that $j_{i}$ appears in a different layer of the system, we will set up a similar equivalence in that layer, and use the same $b_{i}$ at both places.

- Now, we have to handle the extended vectors appearing in the context after the above extensions. They are $\widehat{\mathbf{v}}_{1}=\operatorname{ext}\left(j_{1}, \mathbf{v}_{1}^{*}\right), \ldots, \widehat{\mathbf{v}}_{\ell-1}=\operatorname{ext}\left(j_{\ell-1}, \mathbf{v}_{\ell-1}^{*}\right) \in$ $\{0,1\}^{4 n k}$ and $\widehat{\mathbf{w}}_{1}=\operatorname{ext}\left(\bar{j}_{1}, \mathbf{w}_{1}^{*}\right), \ldots, \widehat{\mathbf{w}}_{\ell}=\operatorname{ext}\left(\bar{j}_{\ell}, \mathbf{w}_{\ell}^{*}\right) \in\{0,1\}^{4 n k}$, as well as $\widehat{\mathbf{p}}=\operatorname{ext}\left(j_{\ell}, \mathbf{p}^{*}\right) \in\{0,1\}^{4 n k-2}$. To prove that these vector are well-formed, we will use the following permuting techniques.

Let $r \in\{2 n k, 2 n k-1\}$. For $b \in\{0,1\}$ and $\pi \in \mathcal{S}_{r}$, we define the permutation $F_{b, \pi}$ that transforms $\mathbf{t}=\left(\begin{array}{c}\mathbf{t}_{0} \\ \mathbf{t}_{1}\end{array}\right) \in \mathbb{Z}^{2 r}$ consisting of 2 blocks of size $r$ into 
$F_{b, \pi}(\mathbf{t})=\left(\begin{array}{c}\pi\left(\mathbf{t}_{b}\right) \\ \pi\left(\mathbf{t}_{\bar{b}}\right)\end{array}\right)$. Namely, $F_{b, \pi}$ first rearranges the blocks of $\mathbf{t}$ according to $b$ (it keeps the arrangement of blocks if $b=0$, or swaps them if $b=1$ ), then it permutes each block according to $\pi$. Note that, we have the following, for all $b_{1}, \ldots, b_{\ell} \in\{0,1\}, \phi_{v, 1}, \ldots, \phi_{v, \ell-1}, \phi_{w, 1}, \ldots, \phi_{w, \ell} \in \mathcal{S}_{2 n k}$, and $\pi_{p} \in \mathcal{S}_{2 n k-1}:$

$$
\left\{\begin{array}{l}
\forall i \in[\ell-1]: \widehat{\mathbf{v}}_{i}=\operatorname{ext}\left(j_{i}, \mathbf{v}_{i}^{*}\right) \Longleftrightarrow F_{b_{i}, \phi_{v, i}}\left(\widehat{\mathbf{v}}_{i}\right)=\operatorname{ext}\left(j_{i} \oplus b_{i}, \phi_{v, i}\left(\mathbf{v}_{i}^{*}\right)\right) ; \\
\forall i \in[\ell]: \widehat{\mathbf{w}}_{i}=\operatorname{ext}\left(\bar{j}_{i}, \mathbf{w}_{i}^{*}\right) \Longleftrightarrow F_{b_{i}, \phi_{w, i}}\left(\widehat{\mathbf{w}}_{i}\right)=\operatorname{ext}\left(\bar{j}_{i} \oplus b_{i}, \phi_{w, i}\left(\mathbf{w}_{i}^{*}\right)\right) ; \\
\widehat{\mathbf{p}}=\operatorname{ext}\left(j_{\ell}, \mathbf{p}^{*}\right) \Longleftrightarrow F_{b_{\ell}, \pi_{p}}(\widehat{\mathbf{p}})=\operatorname{ext}\left(j_{\ell} \oplus b_{\ell}, \pi_{p}\left(\mathbf{p}^{*}\right)\right)
\end{array}\right.
$$

Combining these permuting techniques with the ones described earlier, we can prove that the considered extended vectors are well-formed, with respect to the secret $j_{1}, \ldots, j_{\ell}, \mathbf{v}_{1}^{*}, \ldots, \mathbf{v}_{\ell-1}^{*}, \mathbf{w}_{1}^{*}, \ldots, \mathbf{w}_{\ell}^{*}, \mathbf{p}^{*}$.

Given the above transformations, we now unify all the secret objects into vector $\mathbf{z} \in\{0,1\}^{D}$, where $D=10 n k \ell+2 m+4 m_{E}+2 \ell-3$, of the following form (we abuse the notation of transposition):

$$
\left(\mathbf{v}_{1}^{*}\left\|\widehat{\mathbf{v}}_{1}\right\| \widehat{\mathbf{w}}_{1}\|\ldots\| \mathbf{v}_{\ell-1}^{*}\left\|\widehat{\mathbf{v}}_{\ell-1}\right\| \widehat{\mathbf{w}}_{\ell-1}\left\|\mathbf{p}^{*}\right\| \widehat{\mathbf{p}}\left\|\widehat{\mathbf{w}}_{\ell}\right\| \mathbf{x}^{*}\left\|\mathbf{r}_{1}^{*}\right\| \mathbf{r}_{2}^{*}\left\|\mathbf{j}_{1}\right\| \ldots \| \mathbf{j}_{\ell}\right) .
$$

Then, we observe that, the equations in (8) can be equivalently combined into one equation $\mathbf{M} \cdot \mathbf{z}=\mathbf{u} \bmod q$, where matrix $\mathbf{M}$ and vector $\mathbf{u}$ are built from the public input.

Next, to apply the permuting techniques we have discussed above, let us define VALID as the set of all vectors in $\{0,1\}^{D}$, that have the form (9), where $\mathbf{x}^{*} \in \mathrm{B}_{m}^{2 m}, \mathbf{r}_{1}^{*}, \mathbf{r}_{2}^{*} \in \mathrm{B}_{m_{E}}^{2 m_{E}}, \mathbf{p}^{*} \in \mathrm{B}_{n k}^{2 n k-1}, \mathbf{v}_{1}^{*}, \ldots, \mathbf{v}_{\ell-1}^{*} \in \mathrm{B}_{n k}^{2 n k}$, and there exist $j_{1}, \ldots, j_{\ell} \in\{0,1\}, \mathbf{w}_{1}^{*}, \ldots, \mathbf{w}_{\ell}^{*} \in \mathrm{B}_{n k}^{2 n k}$, such that:

$$
\left\{\begin{array}{l}
\widehat{\mathbf{p}}=\operatorname{ext}\left(j_{\ell}, \mathbf{p}^{*}\right), \quad \forall i \in[\ell]: \mathbf{j}_{i}=\operatorname{ext}_{2}\left(j_{i}\right) \\
\forall i \in[\ell-1]: \widehat{\mathbf{v}}_{i}=\operatorname{ext}\left(j_{i}, \mathbf{v}_{i}^{*}\right), \quad \forall i \in[\ell]: \widehat{\mathbf{w}}_{i}=\operatorname{ext}\left(\bar{j}_{i}, \mathbf{w}_{i}^{*}\right)
\end{array}\right.
$$

It can be seen that our vector $\mathbf{z}$ belongs to this tailored set VALID. To prove that $\mathbf{z} \in$ VALID using random permutations, let us determine how to permute the coordinates of $\mathbf{z}$. To this end, we first define the set:

$$
\overline{\mathcal{S}}=\{0,1\}^{\ell} \times \mathcal{S}_{2 m} \times \mathcal{S}_{2 n k-1} \times\left(\mathcal{S}_{2 m_{E}}\right)^{2} \times\left(\mathcal{S}_{2 n k}\right)^{2 \ell-1} .
$$

Then, $\forall \eta=\left(\left(b_{1}, \ldots, b_{\ell}\right), \pi_{x}, \pi_{p},\left(\pi_{r, 1}, \pi_{r, 2}\right),\left(\phi_{v, 1}, \ldots, \phi_{v, \ell-1}, \phi_{w, 1}, \ldots, \phi_{w, \ell}\right)\right) \in \overline{\mathcal{S}}$, we let $\Gamma_{\eta}$ be the permutation that, when applying to vector $\mathbf{t} \in \mathbb{Z}^{D}$ whose blocks are as in (9), it transforms those blocks as follows:

- For all $i \in[\ell-1]: \quad \mathbf{v}_{i}^{*} \mapsto \phi_{v, i}\left(\mathbf{v}_{i}^{*}\right) ; \quad \widehat{\mathbf{v}}_{i} \mapsto F_{b_{i}, \phi_{v, i}}\left(\widehat{\mathbf{v}}_{i}\right)$.

- For all $i \in[\ell]: \quad \widehat{\mathbf{w}}_{i} \mapsto F_{b_{i}, \phi_{w, i}}\left(\widehat{\mathbf{w}}_{i}\right) ; \quad \mathbf{j}_{i} \mapsto T_{b_{i}}\left(\mathbf{j}_{i}\right)$.

$-\mathbf{p}^{*} \mapsto \pi_{p}\left(\mathbf{p}^{*}\right) ; \quad \widehat{\mathbf{b}} \mapsto F_{b_{\ell}, \pi_{p}}(\widehat{\mathbf{p}})$.

$-\mathbf{x}^{*} \mapsto \pi_{x}\left(\mathbf{x}^{*}\right) ; \quad \mathbf{r}_{1}^{*} \mapsto \pi_{r, 1}\left(\mathbf{r}_{1}^{*}\right) ; \quad \mathbf{r}_{2}^{*} \mapsto \pi_{r, 2}\left(\mathbf{r}_{2}^{*}\right)$. 
It now can be checked that we have the desired equivalence: For all $\eta \in \overline{\mathcal{S}}$,

$$
\mathbf{z} \in \text { VALID } \Longleftrightarrow \Gamma_{\eta}(\mathbf{z}) \in \text { VALID. }
$$

At this point, we can now run a simple Stern-like protocol to prove knowledge of $\mathbf{z} \in$ VALID such that $\mathbf{M} \cdot \mathbf{z}=\mathbf{u} \bmod q$. The common input is the pair $(\mathbf{M}, \mathbf{u})$, while the prover's secret input is $\mathbf{z}$. The interaction between prover $\mathcal{P}$ and verifier $\mathcal{V}$ is described in Fig. 3. The protocol employs the string commitment scheme COM from [24 that is statistically hiding and computationally binding if the $\operatorname{SIVP}_{\widetilde{\mathcal{O}}(n)}$ problem is hard.

1. Commitment: Prover samples $\mathbf{r}_{z} \stackrel{\$}{\leftarrow} \mathbb{Z}_{q}^{D}, \eta \stackrel{\$}{\leftarrow}$ and randomness $\rho_{1}, \rho_{2}, \rho_{3}$ for COM. Then he sends CMT $=\left(C_{1}, C_{2}, C_{3}\right)$ to the verifier, where

$$
C_{1}=\operatorname{CoM}\left(\eta, \mathbf{M} \cdot \mathbf{r}_{z} ; \rho_{1}\right), \quad C_{2}=\operatorname{COM}\left(\Gamma_{\eta}\left(\mathbf{r}_{z}\right) ; \rho_{2}\right), \quad C_{3}=\operatorname{COM}\left(\Gamma_{\eta}\left(\mathbf{z}+\mathbf{r}_{z}\right) ; \rho_{3}\right) .
$$

2. Challenge: The verifier sends a challenge $C h \stackrel{\$}{\leftarrow}\{1,2,3\}$ to the prover.

3. Response: Depending on $C h$, the prover sends RSP computed as follows:

- $C h=1$ : Let $\mathbf{t}_{z}=\Gamma_{\eta}(\mathbf{z}), \mathbf{t}_{r}=\Gamma_{\eta}\left(\mathbf{r}_{z}\right)$, and $\operatorname{RSP}=\left(\mathbf{t}_{z}, \mathbf{t}_{r}, \rho_{2}, \rho_{3}\right)$.

- Ch $=2$ : Let $\eta_{2}=\eta, \mathbf{z}_{2}=\mathbf{z}+\mathbf{r}_{z}$, and RSP $=\left(\eta_{2}, \mathbf{z}_{2}, \rho_{1}, \rho_{3}\right)$.

- $C h=3$ : Let $\eta_{3}=\eta, \mathbf{z}_{3}=\mathbf{r}_{z}$, and $\operatorname{RSP}=\left(\eta_{3}, \mathbf{z}_{3}, \rho_{1}, \rho_{2}\right)$.

Verification: Receiving RSP, the verifier proceeds as follows:

- $C h=1$ : Check that $\mathbf{t}_{z} \in \mathrm{VALID}$ and $C_{2}=\operatorname{COM}\left(\mathbf{t}_{r} ; \rho_{2}\right), C_{3}=\operatorname{COM}\left(\mathbf{t}_{z}+\mathbf{t}_{r} ; \rho_{3}\right)$.

- $C h=2$ : Check that $C_{1}=\operatorname{COM}\left(\eta_{2}, \mathbf{M} \cdot \mathbf{z}_{2}-\mathbf{u} ; \rho_{1}\right), C_{3}=\operatorname{COM}\left(\Gamma_{\eta_{2}}\left(\mathbf{z}_{2}\right) ; \rho_{3}\right)$.

$-C h=3$ : Check that $C_{1}=\operatorname{COM}\left(\eta_{3}, \mathbf{M} \cdot \mathbf{z}_{3} ; \rho_{1}\right), C_{2}=\operatorname{COM}\left(\Gamma_{\eta_{3}}\left(\mathbf{z}_{3}\right) ; \rho_{2}\right)$.

In each case, the verifier outputs 1 if and only if all the conditions hold.

Fig. 3: Our Stern-like zero-knowledge argument of knowledge.

Theorem 2. Assume that the $\mathrm{SIVP}_{\widetilde{\mathcal{O}}(n)}$ problem is hard. Then the protocol in Fig. [3 is a statistical ZKAoK with perfect completeness, soundness error $2 / 3$, and communication cost $\widetilde{\mathcal{O}}(D \log q)$. Namely:

- There exists a polynomial-time simulator that, on input $(\mathbf{M}, \mathbf{u})$, outputs an accepted transcript statistically close to that produced by the real prover.

- There exists a polynomial-time knowledge extractor that, on input a commitment $\mathrm{CMT}$ and 3 valid responses $\left(\mathrm{RSP}_{1}, \mathrm{RSP}_{2}, \mathrm{RSP}_{3}\right)$ to all 3 possible values of the challenge Ch, outputs $\mathbf{z}^{\prime} \in \mathrm{VALID}$ such that $\mathbf{M} \cdot \mathbf{z}^{\prime}=\mathbf{u} \bmod q$.

Given vector $\mathbf{z}^{\prime}$ outputted by the knowledge extractor, we can compute a tuple $\zeta^{\prime}$ satisfying the conditions described at the beginning of this subsection, simply by "backtracking" the transformation steps we have done on the way. The protocol has communication cost $\widetilde{\mathcal{O}}\left(\left(10 n k \ell+2 m+4 m_{E}+2 \ell-3\right) \log q\right)=\widetilde{\mathcal{O}}(\lambda \cdot \ell)$ bits.

The proof of Theorem 2 employs standard simulation and extraction techniques for Stern-like protocols 24135|36|28] and is deferred to Appendix A 


\subsection{Zero-Knowledge Argument of Correct Decryption}

We now present the underlying ZKAoK used by the tracing manager to generate $\Pi_{\text {trace }}$. The protocol allows the prover to prove knowledge of decryption keys for the multi-bit version of Regev's encryption scheme, as well as prove the correctness of decryption. While the argument system in Section 4.3 deals only with $\{0,1\}$ witness vectors, here, we have to handle secret vectors/matrices of infinity norm larger than 1 . To this end, we will employ the decomposition technique from [35]28] to transform them to vectors with infinity norm 1 . We thus first recall this technique.

Decompositions. For any $B \in \mathbb{Z}_{+}$, define the number $\delta_{B}:=\left\lfloor\log _{2} B\right\rfloor+1=$ $\left\lceil\log _{2}(B+1)\right\rceil$ and the sequence $B_{1}, \ldots, B_{\delta_{B}}$, where $B_{j}=\left\lfloor\frac{B+2^{j-1}}{2^{j}}\right\rfloor, \forall j \in\left[1, \delta_{B}\right]$. As observed in [35], the sequence satisfies $\sum_{j=1}^{\delta_{B}} B_{j}=B$ and any integer $v \in$ $[-B, B]$ can be decomposed into vector $\left(v^{(1)}, \ldots, v^{\left(\delta_{B}\right)}\right)^{\top} \in\{-1,0,1\}^{\delta_{B}}$ such that $\sum_{j=1}^{\delta_{B}} B_{j} \cdot v^{(j)}=v$.

Next, for any positive integers $\mathfrak{m}, B$, we define the decomposition matrix:

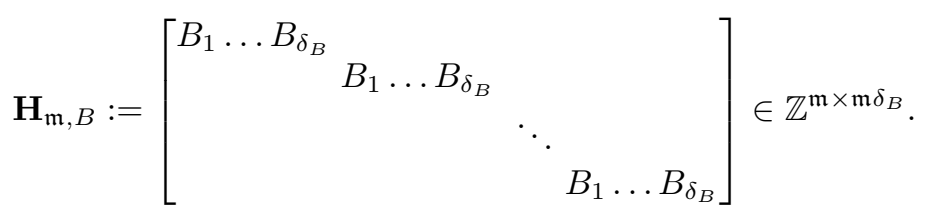

It then follows that, any vector $\mathbf{v} \in[-B, B]^{\mathfrak{m}}$ can be decomposed into vector $\overline{\mathbf{v}} \in\{-1,0,1\}^{\mathfrak{m} \delta_{B}}$ such that $\mathbf{H}_{\mathfrak{m}, B} \cdot \overline{\mathbf{v}}=\mathbf{v}$.

Once we have obtained a transformed witness vector $\overline{\mathbf{v}} \in\{-1,0,1\}^{\mathfrak{m} \delta_{B}}$, we then employ the usual extending-permuting technique for Stern-like protocols. For any positive integer $i$, we let $\mathrm{R}_{i}^{3}$ be the set of all vectors in $\{-1,0,1\}^{3 i}$ that have exactly $i$ coordinates equal to $j$, for each $j \in\{-1,0,1\}$. Note that this set is "closed" under permutations, i.e., for any $\pi \in \mathcal{S}_{3 i}$ and any vector $\mathbf{x} \in \mathrm{R}_{i}^{3}$, the following equivalence holds:

$$
\mathbf{x} \in \mathrm{R}_{i}^{3} \Leftrightarrow \pi(\mathbf{x}) \in \mathrm{R}_{i}^{3} .
$$

The protocol is summarized as follows.

Public input: $\mathbf{B} \in \mathbb{Z}_{q}^{n \times m_{E}}, \mathbf{P}_{1} \in \mathbb{Z}_{q}^{\ell \times m_{E}}, \mathbf{c}_{1,1} \in \mathbb{Z}_{q}^{n}, \mathbf{c}_{1,2} \in \mathbb{Z}_{q}^{\ell}, \mathbf{b}^{\prime} \in\{0,1\}^{\ell}$.

Prover's goal: Proving knowledge of $\mathbf{S}_{1} \in[-\beta, \beta]^{n \times \ell}, \mathbf{E}_{1} \in[-\beta, \beta]^{\ell \times m_{E}}$, and $\mathbf{y} \in[-\lceil q / 5\rceil,\lceil q / 5\rceil]^{\ell}$, such that:

$$
\left\{\begin{array}{l}
\mathbf{S}_{1}^{\top} \cdot \mathbf{B}+\mathbf{E}_{1}=\mathbf{P}_{1} \bmod q ; \\
\mathbf{c}_{1,2}-\mathbf{S}_{1}^{\top} \cdot \mathbf{c}_{1,1}=\mathbf{y}+\lfloor q / 2\rfloor \cdot \mathbf{b}^{\prime} \bmod q .
\end{array}\right.
$$

For each $j \in[\ell]$, let $\mathbf{s}_{j}, \mathbf{e}_{j}, \mathbf{p}_{j}$ be the $j$-th column of matrix $\mathbf{S}_{1}, \mathbf{E}_{1}^{\top}, \mathbf{P}_{1}^{\top}$, respectively; and let $y_{j}, c_{j}, b_{j}$ be the $j$-th entry of vector $\mathbf{y}, \mathbf{c}_{1,2}, \mathbf{b}^{\prime}$, respectively. Then observe that (11) can be re-written as:

$$
\forall j \in[\ell]:\left\{\begin{array}{l}
\mathbf{B}^{T} \cdot \mathbf{s}_{j}+\mathbf{I}_{m_{E}} \cdot \mathbf{e}_{j}=\mathbf{p}_{j} \bmod q \\
\mathbf{c}_{1,1}^{T} \cdot \mathbf{s}_{j}+1 \cdot y_{j}=c_{j}-b_{j} \cdot\lfloor q / 2\rfloor \bmod q .
\end{array}\right.
$$


Using basic algebra, we can manipulate the equations in (12) by rearranging the secret vectors and forming public matrices accordingly. As a result, we obtain a unifying equation of the form

$$
\mathbf{M}_{0} \cdot \mathbf{z}_{0}=\mathbf{u} \bmod q,
$$

where $\mathbf{M}_{0}, \mathbf{u}$ are built from the public input while $\mathbf{z}_{0}=\left(\mathbf{z}_{1} \| \mathbf{z}_{2}\right)$, with

$$
\left\{\begin{array}{l}
\mathbf{z}_{1}=\left(\mathbf{s}_{1}\|\cdots\| \mathbf{s}_{\ell}\left\|\mathbf{e}_{1}\right\| \cdots \| \mathbf{e}_{\ell}\right) \in[-\beta, \beta]^{\left(n+m_{E}\right) \ell} \\
\mathbf{z}_{2}=\left(y_{1}, \ldots, y_{\ell}\right)^{\top} \in[\lceil-q / 5\rceil,\lceil q / 5\rceil]^{\ell}
\end{array}\right.
$$

Next, we can use the decomposition and extension techniques described above to handle our secret vectors. First, decompose $\mathbf{z}_{1}$ into $\overline{\mathbf{z}}_{1} \in\{-1,0,1\}^{D_{1}}$ with $D_{1}=\left(n+m_{E}\right) \ell \delta_{\beta}$ and $\mathbf{z}_{2}$ into $\overline{\mathbf{z}}_{2} \in\{-1,0,1\}^{D_{2}}$ with $D_{2}=\ell \delta_{\lceil q / 5\rceil}$, and form the secret vector $\overline{\mathbf{z}}_{0}=\left(\overline{\mathbf{z}}_{1} \| \overline{\mathbf{z}}_{2}\right) \in\{-1,0,1\}^{L}$ with $D^{\prime}=D_{1}+D_{2}$. Then extend $\overline{\mathbf{z}}_{0}$ to $\mathbf{z} \in \mathrm{R}_{D^{\prime}}^{3}$. Using some basic algebra, we can form public matrix $\mathbf{M}$ such that

$$
\mathbf{M} \cdot \mathbf{z}=\mathbf{M}_{0} \cdot \mathbf{z}_{0}=\mathbf{u} \bmod q .
$$

Now, define the tailored set VALID $=\mathrm{R}_{D^{\prime}}^{3}$ and let $\overline{\mathcal{S}}=\mathcal{S}_{D}$ with $D=3 D^{\prime}$. For every $\eta \in \overline{\mathcal{S}}$, define $\Gamma_{\eta}=\eta$. It is checked that our secret vector $\mathbf{z} \in$ VALID. Observe the equivalence in (10), for any $\eta \in \overline{\mathcal{S}}$, the following equivalence also holds:

$$
\mathbf{z} \in \mathrm{VALID} \Longleftrightarrow \Gamma_{\eta}(\mathbf{z}) \in \mathrm{VALID}
$$

At this point, we can see that the desired statistical ZKAoK protocol can be obtained by running the interactive protocol described in Fig. 3.

\section{Achieving Deniability with Ease}

This section presents how to make our fully dynamic group signature scheme deniable, in the sense of 22 and in a relatively simple manner. In Section 5.1, we first incorporate Ishida et al.'s notion of deniability into Bootle et al.'s model [7]. Then, we show in Section 5.2 that a scheme satisfying this extended model can be easily constructed based on the one from Section 4 and a zero-knowledge argument of "denial opening", demonstrating that a given Regev ciphertext does not decrypt to a particular message. The zero-knowledge argument is described in detail in Section 5.3.

\subsection{Fully Dynamic Group Signatures with Deniability}

We now extend Bootle et al.'s model of FDGS (in Section 2.2) to capture the notion of deniability. Our extension follows Ishida et al.'s approach [22] when they incorporate the deniability functionality into Sakai et al.'s model [47].

A fully dynamic group signature scheme with deniability (FDGSwD) consists of all algorithms of an FDGS, as specified in Section 2.2. and two additional algorithms: DTrace and DJudge. 
DTrace(gpk, tsk, info $_{\tau}$, reg, uid' $\left., M, \Sigma\right) \rightarrow \Pi_{D\left(\text { uid' }^{\prime}\right)}$. The denial opening algorithm, run by the TM, returns a proof $\Pi_{D\left(\text { uid' }^{\prime}\right)}$ showing that uid' did not compute the signature $\Sigma$ on the message $M$ at time $\tau$.

DJudge(gpk, uid, info $\left.{ }_{\tau}, \Pi_{D\left(\text { uid' }^{\prime}\right)}, M, \Sigma\right) \rightarrow 0 / 1$. The denial judgement algorithm checks the validity of $\Pi_{D \text { (uid') }}$ outputted by the DTrace algorithm.

An FDGSwD scheme must satisfy correctness, anonymity, non-frameability, traceability and tracing soundness. To formalize these requirements, we first specify an additional oracle, called DTrace.

DTrace $\left(\right.$ uid $^{\prime}, M, \Sigma$, info $\left._{\tau}\right)$. This oracle returns a denial opening proof $\Pi_{D\left(\text { uid }^{\prime}\right)}$. It is required that $\Sigma$ is not generated by the challenged oracle or the user uid is not in the challenged user set to eliminate the cases where the adversary trivially breaks anonymity.

Then, the correctness and security requirements of an FDGSwD scheme are defined by experiments $\operatorname{Exp}_{\mathrm{FDGSwD}, \mathcal{A}}^{\mathrm{corr}}(\lambda)$ and

$$
\operatorname{Exp}_{\mathrm{FDGSWD}, \mathcal{A}}^{\text {anon-b }}(\lambda), \operatorname{Exp}_{\mathrm{FDGSwD}, \mathcal{A}}^{\text {non-frame }}(\lambda), \operatorname{Exp}_{\mathrm{FDGSwD}, \mathcal{A}}^{\text {trace }}(\lambda), \operatorname{Exp}_{\mathrm{FDGSwD}, \mathcal{A}}^{\text {trace }- \text { sound }}(\lambda),
$$

described in Fig. 4, 5, and 6. The differences between these requirements and the original ones of Bootle et al.'s model (Section 2.2) are highlighted in the below.

Correctness additionally requires that a (denial) proof generated by algorithm DTrace, with respect to a message-signature pair $(M, \Sigma)$ and an arbitrary user uid' who is not the real signer, is always accepted by algorithm DJudge.

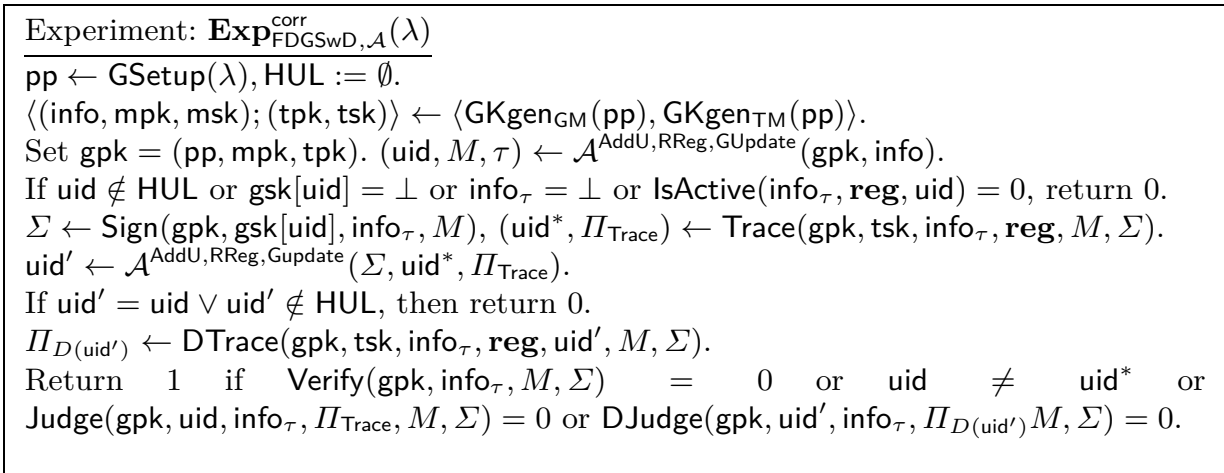

Fig. 4: Experiment to define correctness for an FDGSwD scheme.

Anonymity is defined with respect to an adversary who also has access to the additional oracle DTrace specified above.

Non-frameability additionally requires that it is infeasible for the adversary to generate a valid signature whose denial opening proof with respect to an honest user is not accepted by the DJudge algorithm. 


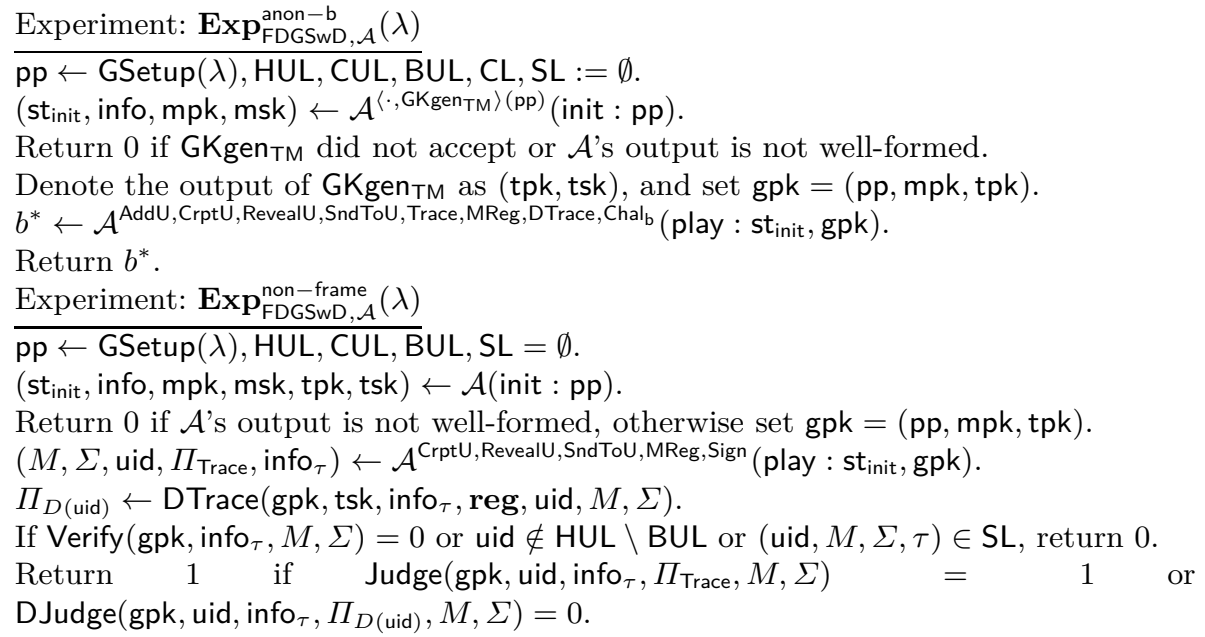

Fig. 5: Experiment to anonymity and non-frameability of an FDGSwD scheme.

Traceability additionally demands that the adversary is not able to produce a valid signature such that opening proof is accepted by the Judge algorithm, but the denial opening proof with respect to the same user is also accepted by the DJudge algorithm.

Tracing Soundness also requires that the adversary should not be able to generate a valid signature such that the denial opening proof for the actual signer is accepted by the DJudge algorithm.

Definition 6. Let $\lambda$ be any security parameter and $\mathcal{A}$ be any PPT adversary. For correctness, non-frameability, traceability and tracing soundness, the advantage of the adversary is defined as the probability of outputting 1 in the corresponding experiment. For anonymity, the advantage is defined as the absolute difference of probability of outputting 1 between experiment $\operatorname{Exp}_{\mathrm{FDGSwD}, \mathcal{A}}^{\mathrm{anon}-1}$ and experiment $\operatorname{Exp}_{\mathrm{FDGSwD}, \mathcal{A}}^{\text {anon-0 }}$.

A fully dynamic group signature scheme with deniability is said to be correct and secure (i.e., anonymous, non-frameable, traceable and tracing sound) if the advantages of the adversary in all considered experiments are negligible in $\lambda$.

\subsection{Our Construction}

As shown by Ishida et al. 22, the main difficulty towards realizing the deniability functionality is to prove in zero-knowledge that a given ciphertext does not decrypt to a particular message. Such a mechanism is non-trivial to realize in general, but it can be done quite easily for our scheme by using the Stern-like technique for proving inequality. Let us look at the equation

$$
\mathbf{c}_{1,2}-\mathbf{S}_{1}^{\top} \cdot \mathbf{c}_{1,1}=\mathbf{y}+\lfloor q / 2\rfloor \cdot \mathbf{b}^{\prime} \bmod q,
$$




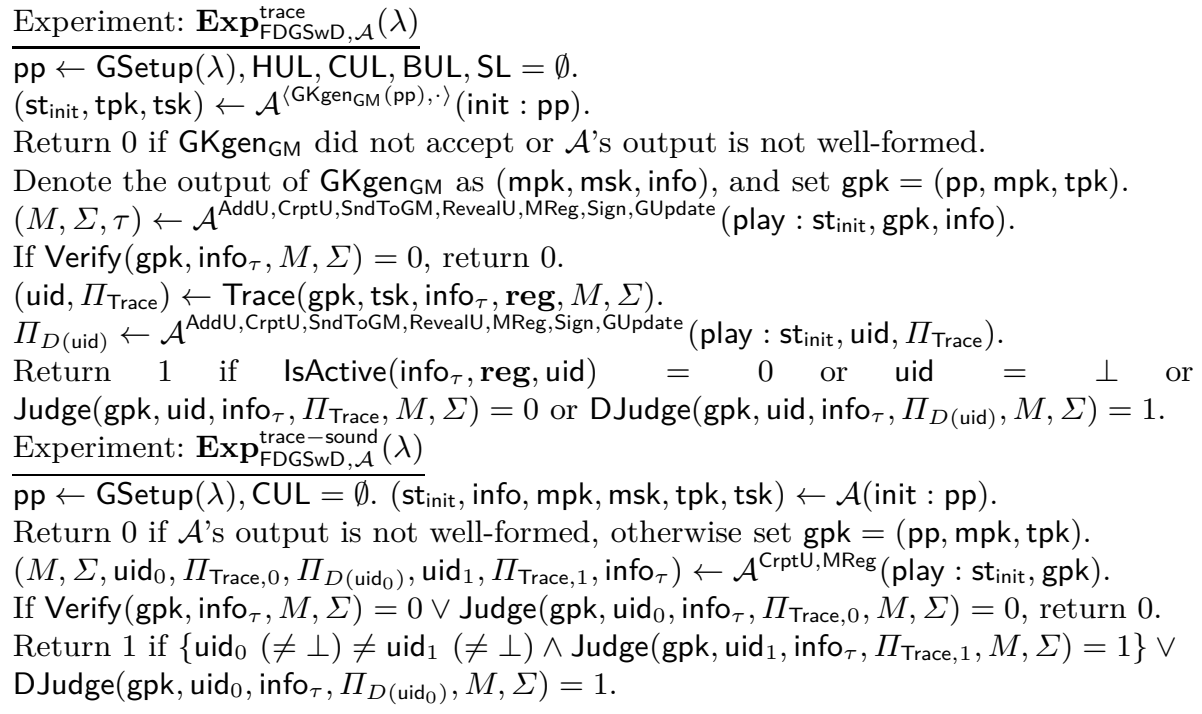

Fig. 6: Experiments to define traceability and tracing soundness of an FDGSwD scheme.

that appears in the argument of correct decryption from Section 4.4. To realize deniability, the plaintext $\mathbf{b}^{\prime} \in\{0,1\}^{\ell}$ should be hidden, and we have to prove that $\mathbf{b}^{\prime} \neq$ uid $^{\prime}$, for some given uid' $\in\{0,1\}^{\ell}$. Subtracting both sides of the equation by $\lfloor q / 2\rfloor \cdot$ uid $^{\prime}$, we get the following:

$$
\mathbf{c}_{1,2}-\mathbf{S}_{1}^{\top} \cdot \mathbf{c}_{1,1}-\lfloor q / 2\rfloor \cdot \mathbf{u i d}^{\prime}=\mathbf{y}+\lfloor q / 2\rfloor \cdot \mathbf{b} \bmod q,
$$

where $\mathbf{b}=\mathbf{b}^{\prime}-$ uid $^{\prime} \in\{-1,0,1\}^{\ell}$ is non-zero. Therefore, the problem towards realizing the deniability is reduced to proving knowledge of a non-zero vector $\mathbf{b} \in\{-1,0,1\}^{\ell}$. This can be done by just appending $2 \ell-1$ "dummy" entries to $\mathbf{b}$ and randomly permuting the extended vector. We defer the detailed description to Section 5.3 .

Now, let us describe and analyze our fully dynamic group signature scheme with deniability. Besides all the algorithms specified in Section 4.1 the scheme contains two additional algorithms, DTrace and DJudge, as follows.

DTrace(gpk, tsk, info ${ }_{\tau}$, reg, uid' $\left.{ }^{\prime}, M, \Sigma\right)$. Let $\mathbf{b}^{\prime}$ be the decryption result of the Trace algorithm, denote $\mathbf{b}=\mathbf{b}^{\prime}-\mathbf{u i d}^{\prime}$. This algorithm generates a NIZKAoK to demonstrate the possession of $\mathbf{S}_{1} \in \mathbb{Z}^{n \times \ell}, \mathbf{E}_{1} \in \mathbb{Z}^{\ell \times m_{E}}, \mathbf{y} \in \mathbb{Z}^{\ell}$, and nonzero vector $\mathbf{b} \in\{-1,0,1\}^{\ell}$ such that

$$
\left\{\begin{array}{l}
\left\|\mathbf{S}_{1}\right\|_{\infty} \leq \beta ;\left\|\mathbf{E}_{1}\right\|_{\infty} \leq \beta ;\|\mathbf{y}\|_{\infty} \leq\lceil q / 5\rceil ; \\
\mathbf{S}_{1}^{\top} \cdot \mathbf{B}+\mathbf{E}_{1}=\mathbf{P}_{1} \bmod q ; \\
\mathbf{c}_{1,2}-\mathbf{S}_{1}^{\top} \cdot \mathbf{c}_{1,1}-\lfloor q / 2\rfloor \cdot \text { uid }^{\prime}=\mathbf{y}+\lfloor q / 2\rfloor \cdot \mathbf{b} \bmod q .
\end{array}\right.
$$

The detailed description of the protocol is presented in Section 5.3. The protocol is repeated $\kappa=\omega(\log \lambda)$ times to achieve negligible soundness error 
and made non-interactive via the Fiat-Shamir heuristic as a triple $\Pi_{D\left(\text { uid' }^{\prime}\right)}=$ $\left(\left\{\mathrm{CMT}_{i}\right\}_{i=1}^{\kappa}, \mathrm{CH},\{\mathrm{RSP}\}_{i=1}^{\kappa}\right)$, where

$$
\mathrm{CH}=\mathcal{H}_{\mathrm{FS}}\left(\left\{\mathrm{CMT}_{i}\right\}_{i=1}^{\kappa}, \mathrm{gpk}_{\text {info }}, M, \Sigma, \text { uid }^{\prime}\right) \in\{1,2,3\}^{\kappa} .
$$

DJudge(gpk, uid', info $\left.\tau, \Pi_{D\left(\text { uid }^{\prime}\right)}, M, \Sigma\right)$. If Verify algorithm outputs 0 , then this algorithm returns 0 . Otherwise, this algorithm then verifies the argument $\Pi_{D\left(\text { uid }^{\prime}\right)}$ with respect to common input (gpk, info $_{\tau}, M, \Sigma$, uid'), in a similar manner as in algorithm Verify.

If $\Pi_{D\left(\text { uid') }^{\prime}\right)}$ does not verify, return 0 . Otherwise, return 1.

EFFICIENCY. In comparison with the FDGS scheme analyzed in Section 4.2, the extended scheme additionally features the NIZKAoK $\Pi_{D(\text { uid') }}$, which is of bit-size $\widetilde{\mathcal{O}}\left(\ell^{2}+\lambda \cdot \ell\right)$. (Recall that $\lambda$ is the security parameter while $\ell=\log N$.)

Correctness. The correctness of the scheme is analyzed as in Section 4.2, but here we additionally need to prove that algorithm DJudge will accept the proof $\Pi_{D \text { (uid') }}$ for uid' that is not the real signer. Since the tracing manager can correctly identify the signer, he possesses a valid tuple $\left(\mathbf{S}_{1}, \mathbf{E}_{1}, \mathbf{y}, \mathbf{b}\right)$ such that the conditions in (13) hold. Correctness then follows from the perfect completeness of our argument system generating $\Pi_{D \text { (uid') }}$.

Anonymity. It is analyzed as in Lemma 2, except that the challenger additionally needs to answer the DTrace oracle queries. However, this can be done in the same way it answers the Trace oracle queries. In other words, when the challenger replies with real proof $\Pi_{\text {trace }}$, it also replies with real proof $\Pi_{D(\cdot)}$; when the challenger replies with simulated proof $\Pi_{\text {trace }}$, it also replies with simulated proof $\Pi_{D(\cdot)}$. This minor change does not affect anonymity, since the argument system generating $\Pi_{D(\cdot)}$ is statistically zero-knowledge.

Non-Frameability. We prove non-frameability by contradiction. Suppose that the non-frameability adversary $\mathcal{A}$ succeeds with non-negligible advantage $\epsilon$. Then we build a PPT algorithm $\mathcal{B}$ that, with non-negligible probability, either breaks the security of our accumulator or solves $\mathrm{SIS}_{n, q, m, 1}^{\infty}$ problem associated with matrix A.

Given a matrix $\mathbf{A}$ from the environment that $\mathcal{B}$ is in, it first generates all parameters pp as we do in GSetup, then invokes $\mathcal{A}$ with pp, and then proceeds as described in the experiment $\operatorname{Exp}_{\mathrm{FDGSwD}, \mathcal{A}}^{\text {non-frame }}(\lambda)$. Here $\mathcal{B}$ can consistently answer all the oracle queries made by $\mathcal{A}$. When $\mathcal{A}$ outputs $\left(M^{*}, \Sigma^{*}, \operatorname{bin}\left(j^{*}\right), \Pi_{\text {trace }}^{*}\right.$, info $\left._{\tau}\right)$ and wins, $\mathcal{B}$ then proceeds as in Lemma 3 until $\mathcal{B}$ extracts the witness $\zeta^{\prime}=$ $\left(\cdots, \operatorname{bin}\left(j^{\prime}\right), \cdots\right)$. By the correctness of our encryption scheme, $\mathbf{c}_{1}^{*}$ is decrypted to $\operatorname{bin}\left(j^{\prime}\right)$, meaning $\operatorname{bin}\left(j^{\prime}\right)$ is the actual signer of $\Sigma^{*}$. The fact that $\mathcal{A}$ wins implies either case (i) Judge $\left(\mathrm{gpk}, \operatorname{bin}\left(j^{*}\right)\right.$, info $\left._{\tau}, \Pi_{\text {trace }}^{*}, M^{*}, \Sigma^{*}\right)=1$ or case (ii)

$$
\text { DJudge }\left(\mathrm{gpk}, \operatorname{bin}\left(j^{*}\right), \text { info }_{\tau}, \Pi_{D\left(\operatorname{bin}\left(j^{*}\right)\right)}, M^{*}, \Sigma^{*}\right)=0 .
$$

Recall that $\operatorname{bin}\left(j^{*}\right)$ is the framed user outputted by the adversary. We claim that $\operatorname{bin}\left(j^{\prime}\right)=\operatorname{bin}\left(j^{*}\right)$ in both case (i) and case (ii). Lemma 3 proves that the equality holds in case (i). Now we prove it also holds in case (ii). Otherwise, $\operatorname{bin}\left(j^{\prime}\right) \neq$ 
$\operatorname{bin}\left(j^{*}\right)$, i.e., $\Pi_{D\left(\operatorname{bin}\left(j^{*}\right)\right)}$ is a denial proof for user $\operatorname{bin}\left(j^{*}\right)$, who is not the actual signer $\operatorname{bin}\left(j^{\prime}\right)$ of $\Sigma^{*}$. Therefore, by the completeness of the underlying argument system, $\Pi_{D\left(\operatorname{bin}\left(j^{*}\right)\right)}$ will be accepted by the DJudge algorithm, a contradiction of case (ii). Hence, the equality holds in case (ii). To this point, $\mathcal{B}$ proceeds again as in Lemma 3. In the end, $\mathcal{B}$ either breaks the security of our accumulator or solves a SIS $S_{n, m, q, 1}^{\infty}$ instance. Therefore, our FDGSwD scheme satisfies non-frameability.

Traceability. Except for the analysis of Lemma 4 we need to consider an additional case (iii) in which $\mathcal{A}$ wins and prove that this case happens with negligible probability. Case (iii) is the event that $\mathcal{A}$ outputs ( $\left.\operatorname{info}_{\tau}, M, \Sigma\right)$, where $\Sigma=\left(\Pi_{\mathrm{gs}}, \mathbf{c}_{1}, \mathbf{c}_{2}\right)$ is a valid signature; the (honestly generated) opening result is (bin $\left.(j), \Pi_{\text {trace }}\right)$, where $\operatorname{bin}(j)$ is an active user and $\Pi_{\text {trace }}$ is accepted by the Judge algorithm; and then $\mathcal{A}$ outputs a denial proof $\Pi_{D(\operatorname{bin}(j))}$, which is accepted by the DJudge algorithm, after seeing the opening result.

To prove this case happens with negligible probability, we proceed as in Lemma 4 and obtain that $\operatorname{bin}\left(j^{\prime}\right)=\operatorname{bin}(j)$ with overwhelming probability, where $\operatorname{bin}\left(j^{\prime}\right)$ is part of the extracted witness, meaning $\mathbf{c}_{1}, \mathbf{c}_{2}$ are encryptions of $\operatorname{bin}\left(j^{\prime}\right)$. The assumption that $\Pi_{D(\operatorname{bin}(j))}$ will be accepted by the DJudge algorithm implies that $\operatorname{bin}\left(j^{\prime}\right)-\operatorname{bin}(j) \neq \mathbf{0}$ with overwhelming probability, by the soundness of the underlying argument system generating $\Pi_{D(\cdot)}$. This results in a contradiction. Therefore, case (iii) only happens with negligible probability, which implies that our FDGSwD scheme is traceable.

Tracing SOUNDness. Except for the analysis of Lemma 5e need to additionally show that it is infeasible for the adversary to generate a valid signature together with a denial proof (for the actual signer) that is accepted by the DJudge algorithm. In other words, we need to prove the event, when $\mathcal{A}$ outputs $\left(M, \Sigma\right.$, uid $_{0}, \Pi_{\text {trace }, 0}, \Pi_{D\left(\text { uid }_{0}\right)}$, uid $\left._{1}, \Pi_{\left.\text {trace }, 1_{1}, \text { info }_{\tau}\right)}\right)$, both $\Pi_{\text {trace }, 0}$ and $\Pi_{D\left(\text { uid }_{0}\right)}$ are accepted, happens with negligible probability. Parse $\Sigma$ as $\left(\Pi_{\mathrm{gs}},\left(\mathbf{c}_{1,1}, \mathbf{c}_{1,2}\right), \mathbf{c}_{2}\right)$. Since $\Pi_{\text {trace, } 0}$ is a valid opening proof, as in Lemma 5 , we can extract witness $\mathbf{S}_{1}, \mathbf{E}_{1}, \mathbf{y}$ such that $\mathbf{S}_{1}^{\top} \cdot \mathbf{B}+\mathbf{E}_{1}=\mathbf{P}_{1} \bmod q$ and

$$
\mathbf{c}_{1,2}-\mathbf{S}_{1}^{\top} \cdot \mathbf{c}_{1,1}=\mathbf{y}+\lfloor q / 2\rfloor \cdot \operatorname{uid}_{0} \bmod q .
$$

The fact that $\Pi_{D\left(\text { uid }_{0}\right)}$ is accepted by the DJudge algorithm implies decryption of $\left(\mathbf{c}_{1,1}, \mathbf{c}_{1,2}\right)$ is not equal to uid ${ }_{0}$, due to the soundness of the underlying argument system. However, from equation (15)), we know decryption of $\left(\mathbf{c}_{1,1}, \mathbf{c}_{1,2}\right)$ is uid $_{0}$, by correctness of our encryption scheme. This results in a contradiction. Therefore, the considered event occurs only with negligible probability, and the FDGSwD scheme is tracing sound.

\subsection{Zero-Knowledge Argument of "Denial Opening"}

We now present the underlying ZKAoK used by the tracing manager to generate $\Pi_{D(\cdot)}$. The protocol allows the prover to show that a given ciphertext does not decrypt to a particular plaintext. The only difference of this protocol with the one described in Section 4.4 is that we need to additionally prove knowledge of a 
non-zero vector $\mathbf{b} \in\{-1,0,1\}^{\ell}$. To this end, we will use the following extendingpermuting technique for Stern-like protocols, which is developed based on Ling et al.'s idea [35].

For any integer $\mathfrak{m} \in \mathbb{Z}_{+}$, define $\widetilde{\mathrm{R}}_{\mathfrak{m}}^{3}$ to be the set of all vectors in $\{-1,0,1\}^{3 \mathfrak{m}-1}$ that has $\mathfrak{m}$ coordinates equal to -1 and 1 , and $\mathfrak{m}-1$ coordinates equal to 0 . To prove that $\mathbf{b} \in\{-1,0,1\}^{\ell}$ and $\mathbf{b} \neq \mathbf{0}^{\ell}$, we append $2 \ell-1$ "dummy" entries to $\mathbf{b}$ to obtain $\mathbf{b}^{*} \in \widetilde{R}_{\ell}^{3}$. Observe that $\mathbf{b} \in\{-1,0,1\}^{\ell}$ is non-zero if and only if the extended vector $\mathbf{b}^{*} \in \widetilde{\mathrm{R}}_{\ell}^{3}$. Furthermore, for any $\pi_{b} \in \mathcal{S}_{3 \ell-1}$, the following equivalence holds:

$$
\mathbf{b}^{*} \in \widetilde{\mathrm{R}}_{\ell}^{3} \Leftrightarrow \pi_{b}\left(\mathbf{b}^{*}\right) \in \widetilde{\mathrm{R}}_{\ell}^{3} .
$$

Now, by using the same technique described in Section 4.4, we can unify equations in (13) into

$$
\widetilde{\mathbf{M}} \cdot \widetilde{\mathbf{z}}=\widetilde{\mathbf{u}} \bmod q,
$$

where $\widetilde{\mathbf{M}}$ and $\widetilde{\mathbf{u}}$ are built from public input while $\widetilde{\mathbf{z}}=\left(\mathbf{z} \| \mathbf{b}^{*}\right) \in\{-1,0,1\}^{\widetilde{D}}$, where $\mathbf{z} \in \mathrm{R}_{D^{\prime}}^{3}$ as defined in Section $4.4 \mathbf{b}^{*} \in \widetilde{\mathrm{R}}_{\ell}^{3}$, and $\widetilde{D}=D+3 \ell-1$.

Define $\widetilde{\text { VALID }}=\mathrm{R}_{D^{\prime}}^{3} \times \widetilde{\mathrm{R}}_{\ell}^{3}$ and $\widetilde{\mathcal{S}}=\mathcal{S}_{D} \times \mathcal{S}_{3 \ell-1}$. For every $\widetilde{\eta}=\left(\eta, \pi_{b}\right) \in \widetilde{\mathcal{S}}$, define $\Gamma_{\widetilde{\eta}}=\widetilde{\eta}$. It can be seen that our secret vector $\widetilde{\mathbf{z}}$ belongs to this tailed set $\widetilde{\mathrm{VALID}}$ and the following equivalence holds:

$$
\widetilde{\mathbf{z}} \in \widetilde{\operatorname{VALID}} \Longleftrightarrow \Gamma_{\widetilde{\eta}}(\widetilde{\mathbf{z}}) \in \widetilde{\text { VALID }},
$$

for any $\widetilde{\eta} \in \widetilde{\mathcal{S}}$. At this point, we can see that the desired statistical ZKAoK protocol can be obtained from the one in Fig. 3 .

We remark that the above technique for obtaining zero-knowledge argument of "denial opening" is also applicable for several other existing lattice-based group signatures [36|30|28] which employ similar tracing mechanisms as ours.

Acknowledgements. The authors would like to thank Benoit Libert for helpful comments and discussions. The research is supported by Singapore Ministry of Education under Research Grant MOE2016-T2-2-014(S).

\section{References}

1. M. Ajtai. Generating Hard Instances of Lattice Problems (Extended Abstract). In STOC 1996, pages 99-108. ACM, 1996.

2. G. Ateniese, J. Camenisch, M. Joye, and G. Tsudik. A Practical and Provably Secure Coalition-Resistant Group Signature Scheme. In CRYPTO 2000, volume 1880 of Lecture Notes in Computer Science, pages 255-270. Springer, 2000.

3. N. Baric and B. Pfitzmann. Collision-Free Accumulators and Fail-Stop Signature Schemes Without Trees. In EUROCRYPT 1997, volume 1233 of Lecture Notes in Computer Science, pages 480-494. Springer, 1997.

4. M. Bellare, D. Micciancio, and B. Warinschi. Foundations of Group Signatures: Formal Definitions, Simplified Requirements, and a Construction Based on General Assumptions. In EUROCRYPT 2003, volume 2656 of Lecture Notes in Computer Science, pages 614-629. Springer, 2003. 
5. M. Bellare, H. Shi, and C. Zhang. Foundations of Group Signatures: The Case of Dynamic Groups. In CT-RSA 2005, volume 3376 of Lecture Notes in Computer Science, pages 136-153. Springer, 2005.

6. D. Boneh and H. Shacham. Group Signatures with Verifier-local Revocation. In ACM CCCS 2004, pages 168-177. ACM, 2004.

7. J. Bootle, A. Cerulli, P. Chaidos, E. Ghadafi, and J. Groth. Foundations of Fully Dynamic Group Signatures. In ACNS 2016, volume 9696 of Lecture Notes in Computer Science, pages 117-136. Springer, 2016. Full version: https://eprint.iacr.org/2016/368.pdf

8. J. Bootle, A. Cerulli, P. Chaidos, E. Ghadafi, J. Groth, and C. Petit. Short Accountable Ring Signatures Based on DDH. In ESORICS 2015, volume 9326 of Lecture Notes in Computer Science, pages 243-265. Springer, 2015.

9. X. Boyen. Lattice Mixing and Vanishing Trapdoors: A Framework for Fully Secure Short Signatures and More. In PKC 2010, volume 6056 of Lecture Notes in Computer Science, pages 499-517. Springer, 2010.

10. E. Bresson and J. Stern. Efficient Revocation in Group Signatures. In PKC 2001, volume 1992 of Lecture Notes in Computer Science, pages 190-206. Springer, 2001.

11. E. Brickell, D. Pointcheval, S. Vaudenay, and M. Yung. Design Validations for Discrete Logarithm Based Signature Schemes. In PKC 2000, pages 276-292. Springer, 2000.

12. J. Camenisch and A. Lysyanskaya. Dynamic Accumulators and Application to Efficient Revocation of Anonymous Credentials. In CRYPTO 2002, volume 2442 of Lecture Notes in Computer Science, pages 61-76. Springer, 2002.

13. J. Camenisch, G. Neven, and M. Rückert. Fully Anonymous Attribute Tokens from Lattices. In SCN 2012, volume 7485 of Lecture Notes in Computer Science, pages 57-75. Springer, 2012.

14. D. Cash, D. Hofheinz, E. Kiltz, and C. Peikert. Bonsai Trees, or How to Delegate a Lattice Basis. In EUROCRYPT 2010, volume 6110 of Lecture Notes in Computer Science, pages 523-552. Springer, 2010.

15. D. Chaum and E. van Heyst. Group Signatures. In EUROCRYPT 1991, volume 547 of Lecture Notes in Computer Science, pages 257-265. Springer, 1991.

16. S. Cheng, K. Nguyen, and H. Wang. Policy-Based Signature Scheme from Lattices. Des. Codes Cryptography, 81(1):43-74, 2016.

17. C. Delerablée and D. Pointcheval. Dynamic Fully Anonymous Short Group Signatures. In VIETCRYPT 2006, volume 4341 of Lecture Notes in Computer Science, pages 193-210. Springer, 2006.

18. A. Fiat and A. Shamir. How to Prove Yourself: Practical Solutions to Identification and Signature Problems. In CRYPTO 1986, volume 263 of Lecture Notes in Computer Science, pages 186-194. Springer, 1987.

19. C. Gentry, C. Peikert, and V. Vaikuntanathan. Trapdoors for Hard Lattices and New Cryptographic Constructions. In STOC 2008, pages 197-206. ACM, 2008.

20. S. D. Gordon, J. Katz, and V. Vaikuntanathan. A Group Signature Scheme from Lattice Assumptions. In ASIACRYPT 2010, volume 6477 of Lecture Notes in Computer Science, pages 395-412. Springer, 2010.

21. J. Groth. Fully Anonymous Group Signatures without Random Oracles. In ASIACRYPT 2007, volume 4833 of Lecture Notes in Computer Science, pages 164-180. Springer, 2007.

22. A. Ishida, K. Emura, G. Hanaoka, Y. Sakai, and K. Tanaka. Group Signature with Deniability: How to Disavow a Signature. In CANS 2016, volume 10052 of Lecture Notes in Computer Science, pages 228-244, 2016. 
23. A. Kawachi, K. Tanaka, and K. Xagawa. Multi-bit Cryptosystems Based on Lattice Problems. In PKC 2007, volume 4450 of Lecture Notes in Computer Science, pages 315-329. Springer, 2007.

24. A. Kawachi, K. Tanaka, and K. Xagawa. Concurrently Secure Identification Schemes Based on the Worst-Case Hardness of Lattice Problems. In ASIACRYPT 2008, volume 5350 of Lecture Notes in Computer Science, pages 372-389. Springer, 2008.

25. A. Kiayias and M. Yung. Secure Scalable Group Signature with Dynamic Joins and Separable authorities. IJSN, 1(1):24-45, 2006.

26. F. Laguillaumie, A. Langlois, B. Libert, and D. Stehlé. Lattice-Based Group Signatures with Logarithmic Signature Size. In ASIACRYPT 2013, volume 8270 of Lecture Notes in Computer Science, pages 41-61. Springer, 2013.

27. A. Langlois, S. Ling, K. Nguyen, and H. Wang. Lattice-Based Group Signature Scheme with Verifier-Local Revocation. In PKC 2014, volume 8383 of Lecture Notes in Computer Science, pages 345-361. Springer, 2014.

28. B. Libert, S. Ling, F. Mouhartem, K. Nguyen, and H. Wang. Signature Schemes with Efficient Protocols and Dynamic Group Signatures from Lattice Assumptions. In ASIACRYPT 2016, volume 10032 of Lecture Notes in Computer Science, pages 373-403. Springer, 2016.

29. B. Libert, S. Ling, F. Mouhartem, K. Nguyen, and H. Wang. Zero-Knowledge Arguments for Matrix-Vector Relations and Lattice-Based Group Encryption. In ASIACRYPT 2016, volume 10032 of Lecture Notes in Computer Science, pages 101-131, 2016.

30. B. Libert, S. Ling, K. Nguyen, and H. Wang. Zero-Knowledge Arguments for Lattice-Based Accumulators: Logarithmic-Size Ring Signatures and Group Signatures Without Trapdoors. In EUROCRYPT 2016, volume 9666 of Lecture Notes in Computer Science, pages 1-31. Springer, 2016.

31. B. Libert, F. Mouhartem, and K. Nguyen. A Lattice-Based Group Signature Scheme with Message-Dependent Opening. In ACNS 2016, volume 9696 of Lecture Notes in Computer Science, pages 137-155. Springer, 2016.

32. B. Libert, T. Peters, and M. Yung. Group Signatures with Almost-for-Free Revocation. In CRYPTO 2012, volume 7417 of Lecture Notes in Computer Science, pages 571-589. Springer, 2012.

33. B. Libert, T. Peters, and M. Yung. Scalable Group Signatures with Revocation. In EUROCRYPT 2012, volume 7237 of Lecture Notes in Computer Science, pages 609-627. Springer, 2012.

34. B. Libert, T. Peters, and M. Yung. Short Group Signatures via StructurePreserving Signatures: Standard Model Security from Simple Assumptions. In CRYPTO 2015, volume 9216 of Lecture Notes in Computer Science. Springer, 2015.

35. S. Ling, K. Nguyen, D. Stehlé, and H. Wang. Improved Zero-Knowledge Proofs of Knowledge for the ISIS Problem, and Applications. In PKC 2013, volume 7778, pages 107-124. Springer, 2013.

36. S. Ling, K. Nguyen, and H. Wang. Group Signatures from Lattices: Simpler, Tighter, Shorter, Ring-Based. In PKC 2015, volume 9020 of Lecture Notes in Computer Science, pages 427-449. Springer, 2015.

37. S. Ling, K. Nguyen, H. Wang, and Y. Xu. Lattice-Based Group Signatures: Achieving Full Dynamicity with Ease. In ACNS 2017, volume 10355 of Lecture Notes in Computer Science, pages 293-312. Springer, 2017. 
38. D. Micciancio and P. Mol. Pseudorandom Knapsacks and the Sample Complexity of LWE Search-to-Decision Reductions. In CRYPTO 2011, volume 6841 of Lecture Notes in Computer Science, pages 465-484. Springer, 2011.

39. D. Micciancio and C. Peikert. Hardness of SIS and LWE with Small Parameters. In CRYPTO 2013, volume 8042 of Lecture Notes in Computer Science, pages 21-39. Springer, 2013.

40. T. Nakanishi, H. Fujii, Y. Hira, and N. Funabiki. Revocable Group Signature Schemes with Constant Costs for Signing and Verifying. In PKC 2009, volume 5443 of Lecture Notes in Computer Science, pages 463-480. Springer, 2009.

41. M. Naor and M. Yung. Public-Key Cryptosystems Provably Secure against Chosen Ciphertext Attacks. In STOC 1990, pages 427-437. ACM, 1990.

42. L. Nguyen. Accumulators from Bilinear Pairings and Applications. In CT-RSA 2005, volume 3376 of Lecture Notes in Computer Science, pages 275-292. Springer, 2005.

43. L. Nguyen and R. Safavi-Naini. Efficient and Provably Secure Trapdoor-Free Group Signature Schemes from Bilinear Pairings. In ASIACRYPT 2004, volume 3329 of Lecture Notes in Computer Science, pages 372-386. Springer, 2004.

44. P. Q. Nguyen, J. Zhang, and Z. Zhang. Simpler Efficient Group Signatures from Lattices. In PKC 2015, volume 9020 of Lecture Notes in Computer Science, pages 401-426. Springer, 2015.

45. O. Regev. On Lattices, Learning with Errors, Random Linear Codes, and Cryptography. In STOC 2005, pages 84-93. ACM, 2005.

46. Y. Sakai, K. Emura, G. Hanaoka, Y. Kawai, T. Matsuda, and K. Omote. Group Signatures with Message-Dependent Opening. In Pairing 2012, volume 7708 of Lecture Notes in Computer Science, pages 270-294. Springer, 2012.

47. Y. Sakai, J. Schuldt, K. Emura, G. Hanaoka, and K. Ohta. On the Security of Dynamic Group Signatures: Preventing Signature Hijacking. In PKC 2012, volume 7293 of Lecture Notes in Computer Science, pages 715-732. Springer, 2012.

48. P. W. Shor. Polynomial-Time Algorithms for Prime Factorization and Discrete Logarithms on a Quantum Computer. SIAM Journal on Computing, 26(5):14841509, 1997.

49. J. Stern. A New Paradigm for Public Key Identification. IEEE Transactions on Information Theory, 42(6):1757-1768, 1996.

50. G. Tsudik and S. Xu. Accumulating Composites and Improved Group Signing. In ASIACRYPT 2003, volume 2894 of Lecture Notes in Computer Science, pages 269-286. Springer, 2003.

\section{A Proof of Theorem 2}

We first restate Theorem 2 ,

Theorem 3. The protocol in Fig. 3 is a statistical ZKAoK with perfect completeness, soundness error $2 / 3$, and communication cost $\widetilde{\mathcal{O}}(D \log q)$. Namely:

- There exists a polynomial-time simulator that, on input (M, u), outputs an accepted transcript statistically close to that produced by the real prover.

- There exists a polynomial-time knowledge extractor that, on input a commitment CMT and 3 valid responses $\left(\mathrm{RSP}_{1}, \mathrm{RSP}_{2}, \mathrm{RSP}_{3}\right)$ to all 3 possible values of the challenge $C h$, outputs $\mathbf{z}^{\prime} \in \mathrm{VALID}$ such that $\mathbf{M} \cdot \mathbf{z}^{\prime}=\mathbf{u} \bmod q$. 
Proof. It can be checked that the protocol has perfect completeness: If an honest prover follows the protocol, then he always gets accepted by the verifier. It is also easy to see that the communication cost is bounded by $\widetilde{\mathcal{O}}(D \log q)$.

Zero-Knowledge Property. We construct a PPT simulator SIM interacting with a (possibly dishonest) verifier $\widehat{\mathcal{V}}$, such that, given only the public input, SIM outputs with probability negligibly close to $2 / 3$ a simulated transcript that is statistically close to the one produced by the honest prover in the real interaction.

The simulator first chooses a random $\overline{C h} \in\{1,2,3\}$ as a prediction of the challenge value that $\widehat{\mathcal{V}}$ will not choose.

Case $\overline{C h}=1$ : Using basic linear algebra over $\mathbb{Z}_{q}$, SIM computes a vector $\mathbf{z}^{\prime} \in \mathbb{Z}_{q}^{D}$ such that $\mathbf{M} \cdot \mathbf{z}^{\prime}=\mathbf{u} \bmod q$. Next, it samples $\mathbf{r}_{z} \stackrel{\$}{\leftarrow} \mathbb{Z}_{q}^{D}, \eta \stackrel{\$}{\leftarrow} \overline{\mathcal{S}}$, and randomness $\rho_{1}, \rho_{2}, \rho_{3}$ for COM. Then, it sends $\operatorname{CMT}=\left(C_{1}^{\prime}, C_{2}^{\prime}, C_{3}^{\prime}\right)$ to $\widehat{\mathcal{V}}$, where

$$
\begin{gathered}
C_{1}^{\prime}=\operatorname{com}\left(\eta, \mathbf{M} \cdot \mathbf{r}_{z} ; \rho_{1}\right), \\
C_{2}^{\prime}=\operatorname{Com}\left(\Gamma_{\eta}\left(\mathbf{r}_{z}\right) ; \rho_{2}\right), \quad C_{3}^{\prime}=\operatorname{CoM}\left(\Gamma_{\eta}\left(\mathbf{z}^{\prime}+\mathbf{r}_{z}\right) ; \rho_{3}\right) .
\end{gathered}
$$

Receiving a challenge $C h$ from $\widehat{\mathcal{V}}$, the simulator responds as follows:

- If $C h=1$ : Output $\perp$ and abort.

- If $C h=2$ : Send RSP $=\left(\eta, \mathbf{z}^{\prime}+\mathbf{r}_{z}, \rho_{1}, \rho_{3}\right)$.

- If $C h=3:$ Send RSP $=\left(\eta, \mathbf{r}_{z}, \rho_{1}, \rho_{2}\right)$.

Case $\overline{C h}=2$ : SIM samples $\mathbf{z}^{\prime} \stackrel{\$}{\leftarrow}$ VALID, $\mathbf{r}_{z} \stackrel{\$}{\leftarrow} \mathbb{Z}_{q}^{D}, \eta \stackrel{\$}{\leftarrow}$, and randomness $\rho_{1}, \rho_{2}, \rho_{3}$ for COM. Then it sends CMT $=\left(C_{1}^{\prime}, C_{2}^{\prime}, C_{3}^{\prime}\right)$ to $\widehat{\mathcal{V}}$, where

$$
\begin{gathered}
C_{1}^{\prime}=\operatorname{CoM}\left(\eta, \mathbf{M} \cdot \mathbf{r}_{z} ; \rho_{1}\right), \\
C_{2}^{\prime}=\operatorname{Com}\left(\Gamma_{\eta}\left(\mathbf{r}_{z}\right) ; \rho_{2}\right), \quad C_{3}^{\prime}=\operatorname{CoM}\left(\Gamma_{\eta}\left(\mathbf{z}^{\prime}+\mathbf{r}_{z}\right) ; \rho_{3}\right) .
\end{gathered}
$$

Receiving a challenge $C h$ from $\widehat{\mathcal{V}}$, the simulator responds as follows:

- If $C h=1:$ Send RSP $=\left(\Gamma_{\eta}\left(\mathbf{z}^{\prime}\right), \Gamma_{\eta}\left(\mathbf{r}_{z}\right), \rho_{2}, \rho_{3}\right)$.

- If $C h=2$ : Output $\perp$ and abort.

- If $C h=3$ : Send RSP $=\left(\eta, \mathbf{r}_{z}, \rho_{1}, \rho_{2}\right)$.

Case $\overline{C h}=3$ : SIM samples $\mathbf{z}^{\prime} \stackrel{\$}{\leftarrow}$ VALID, $\mathbf{r}_{z} \stackrel{\$}{\leftarrow} \mathbb{Z}_{q}^{D}, \eta \stackrel{\$}{\leftarrow}$, and randomness $\rho_{1}, \rho_{2}, \rho_{3}$ for COM. Then it sends CMT $=\left(C_{1}^{\prime}, C_{2}^{\prime}, C_{3}^{\prime}\right)$ to $\widehat{\mathcal{V}}$, where $C_{2}^{\prime}=$ $\operatorname{CoM}\left(\Gamma_{\eta}\left(\mathbf{r}_{z}\right) ; \rho_{2}\right), C_{3}^{\prime}=\operatorname{CoM}\left(\Gamma_{\eta}\left(\mathbf{z}^{\prime}+\mathbf{r}_{z}\right) ; \rho_{3}\right)$ as in the previous two cases, while

$$
C_{1}^{\prime}=\operatorname{CoM}\left(\eta, \mathbf{M} \cdot\left(\mathbf{z}^{\prime}+\mathbf{r}_{z}\right)-\mathbf{u} ; \rho_{1}\right) .
$$

Receiving a challenge $C h$ from $\widehat{\mathcal{V}}$, it responds as follows:

- If $C h=1$ : Send RSP computed as in the case $(\overline{C h}=2, C h=1)$.

- If $C h=2$ : Send RSP computed as in the case $(\overline{C h}=1, C h=2)$.

- If $C h=3:$ Output $\perp$ and abort. 
We observe that, in every case, since COM is statistically hiding, the distribution of the commitment CMT and the distribution of the challenge $C h$ from $\widehat{\mathcal{V}}$ are statistically close to those in the real interaction. Hence, the probability that the simulator outputs $\perp$ is negligibly close to $1 / 3$. Moreover, one can check that whenever the simulator does not halt, it will provide an accepted transcript, the distribution of which is statistically close to that of the prover in the real interaction. In other words, we have constructed a simulator that can successfully impersonate the honest prover with probability negligibly close to $2 / 3$.

Argument of Knowledge. Suppose that $\operatorname{RSP}_{1}=\left(\mathbf{t}_{z}, \mathbf{t}_{r}, \rho_{2}, \rho_{3}\right), \mathrm{RSP}_{2}=$ $\left(\eta_{2}, \mathbf{z}_{2}, \rho_{1}, \rho_{3}\right), \mathrm{RSP}_{3}=\left(\eta_{3}, \mathbf{z}_{3}, \rho_{1}, \rho_{2}\right)$ are 3 valid responses to the same commitment $\mathrm{CMT}=\left(C_{1}, C_{2}, C_{3}\right)$, with respect to all 3 possible values of the challenge. The validity of these responses implies that:

$$
\left\{\begin{array}{l}
\mathbf{t}_{z} \in \operatorname{VALID} ; C_{1}=\operatorname{COM}\left(\eta_{2}, \mathbf{M} \cdot \mathbf{z}_{2}-\mathbf{u} ; \rho_{1}\right)=\operatorname{COM}\left(\eta_{3}, \mathbf{M} \cdot \mathbf{z}_{3} ; \rho_{1}\right) ; \\
C_{2}=\operatorname{COM}\left(\mathbf{t}_{r} ; \rho_{2}\right)=\operatorname{COM}\left(\Gamma_{\eta_{3}}\left(\mathbf{z}_{3}\right) ; \rho_{2}\right) ; \\
C_{3}=\operatorname{COM}\left(\mathbf{t}_{z}+\mathbf{t}_{r} ; \rho_{3}\right)=\operatorname{COM}\left(\Gamma_{\eta_{2}}\left(\mathbf{z}_{2}\right) ; \rho_{3}\right) .
\end{array}\right.
$$

Since COM is computationally binding, we can deduce that:

$$
\left\{\begin{array}{l}
\mathbf{t}_{z} \in \mathrm{VALID} ; \eta_{2}=\eta_{3} ; \mathbf{t}_{r}=\Gamma_{\eta_{3}}\left(\mathbf{z}_{3}\right) ; \mathbf{t}_{z}+\mathbf{t}_{r}=\Gamma_{\eta_{2}}\left(\mathbf{z}_{2}\right) \bmod q ; \\
\mathbf{M} \cdot \mathbf{z}_{2}-\mathbf{u}=\mathbf{M} \cdot \mathbf{z}_{3} \bmod q .
\end{array}\right.
$$

Since $\mathbf{t}_{z} \in$ VALID, if we let $\mathbf{z}^{\prime}=\left[\Gamma_{\eta_{2}}\right]^{-1}\left(\mathbf{t}_{z}\right)$, then $\mathbf{z}^{\prime} \in$ VALID. Furthermore, we have

$$
\Gamma_{\eta_{2}}\left(\mathbf{z}^{\prime}\right)+\Gamma_{\eta_{2}}\left(\mathbf{z}_{3}\right)=\Gamma_{\phi_{2}}\left(\mathbf{z}_{2}\right) \bmod q,
$$

which implies that $\mathbf{z}^{\prime}+\mathbf{z}_{3}=\mathbf{z}_{2} \bmod q$, and that $\mathbf{M} \cdot \mathbf{z}^{\prime}+\mathbf{M} \cdot \mathbf{z}_{3}=\mathbf{M} \cdot \mathbf{z}_{2} \bmod q$. As a result, we have $\mathbf{M} \cdot \mathbf{z}^{\prime}=\mathbf{u} \bmod q$. This concludes the proof. 